\title{
ELASTICIDADES INGRESO Y CUALITATIVAS EN MÉXICO: UNA APLICACIÓN DEL MODELO DE ASIGNACIÓN DEL GASTO
}

\author{
A. P. Huyser y W. H. SOMERMEyer * \\ Netherland School of Economics
}

1. TEORÍA GENERAL DE LA CONDUCTA DEL CONSUMIDOR

LA TEORía clásica del consumo examina la conducta del consumidor, que gasta una cantidad fija de dinero durante un cierto período, en un número limitado de bienes a precios predeterminados. Los elementos de esta teoría son:

a) un número finito $1,2,3, \ldots, K$, de bienes disponibles

b) precios dados $p_{1}, p_{2}, p_{3}, \ldots, p_{k} ; p_{k}>0 ; k=1,2, \ldots, K$, a los cuales el consumidor puede adquirir, a voluntad, las cantidades: $q_{1}, \ldots, q_{k}, q_{k} \geq 0 ; k=1, \ldots, K$

c) bajo la restricción presupuestal $C$ :

$$
\sum_{k} p_{k} q_{k} \equiv \sum_{k} C_{k} \equiv C
$$

o bien, en notación matricial

$$
p^{\prime} q=C
$$

Donde $p^{\prime}=\left\{p_{1}, \ldots, p_{K}\right\}$ y $q^{\prime}=\left\{q_{1}, \ldots, q_{K}\right\}$

Si suponemos que el consumidor se comporta racionalmente en el sentido de que maximiza su función utilidad:

$$
u=u\left(q^{\prime}\right)
$$

sujeta a la restricción presupuestal (1.1), la función utilidad indica el nivel de satisfacción que el consumidor deriva del consumo de

$$
q^{\prime}=\left\{q_{1}, \ldots, q_{K}\right\}
$$
son

Las condiciones de primer orden para maximizar (1.2) sujeta a (1.1)

$$
u_{k}=\lambda p_{k} \text { para } k=1, \ldots, K
$$

* Traducción del inglés de Adalberto García Rocha. 
donde $u_{k}=\partial u / \partial q_{k}$ y $\lambda$ es un multiplicador de Lagrange. En notación matricial:

$$
u_{q}=\lambda_{p}, \quad \text { donde } u_{q}^{\prime}=\left\{u_{1}, \ldots, u_{k}\right\}
$$

Las $K+1$ ecuaciones (1.1) y (1.3) contienen $K+1$ incógnitas, a saber, $q_{1}, \ldots, q_{k}$ y $\lambda$; por lo tanto, en principio, puede encontrarse una solución. Para que esta solución sea un óptimo $q^{\circ}$, debe satisfacerse la condición de segundo orden. En la expansión de Taylor de $u$ alrededor de una región infinitesimalmente pequeña de $q^{\circ}$, lo anterior significa que:

$$
\begin{gathered}
u\left(q_{1}^{\circ}+d q_{1}, \ldots, q_{K}^{\circ}+d q_{K}\right)-u\left(q_{1}^{\circ}, \ldots,{ }^{\circ} q_{K}\right) \\
=\sum_{k} u_{k}^{\circ} d q_{k}+\frac{1}{2} \sum_{k k} u_{k l}^{\circ} d q_{k} d q_{l}+\text { términos de orden mayor, esto es, }
\end{gathered}
$$

se requiere que

$$
\text { (1.5) } \quad=\frac{1}{2} \sum_{k l} \sum_{k l} d q_{k} d q_{l}<0
$$

donde $u_{k l}=\partial^{2} u /\left(\partial q_{k} \cdot \partial q_{l}\right)$, de tal modo que $\sum_{k} p_{k} q_{k}=\lambda \underset{k}{\Sigma_{k}} u_{k} d q_{k}=0$, y suponiendo convergencia de la expansión de la serie de Taylor.

Para satisfacer la desigualdad (1.5), una condición suficiente es que la matriz $U$ de derivadas de segundo orden $u_{k l}$ sea negativa definida.

Con base en (1.1) y (1.3) podemos evaluar los efectos de cambios en $p$ y $C$ sobre $q^{\circ}$. Diferenciando estas ecuaciones con respecto a $p$ y $C$, obtenemos:

(1.6) $\left[\begin{array}{l}U \\ p^{\prime}\end{array}\right.$

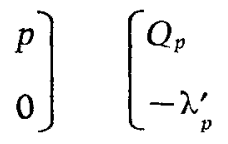

$$
\left.\begin{array}{c}
q_{c} \\
-\lambda_{C}
\end{array}\right]=\left[\begin{array}{l}
\lambda I \\
-q^{\prime}
\end{array}\right.
$$

donde $\quad Q_{p}=\left[\begin{array}{ccc}\partial q_{1} / \partial p_{1} & \ldots & \partial q_{1} / \partial p_{k} \\ \cdot & \cdot \\ \partial q_{k} / \partial p_{1} & \ldots & \partial \dot{q_{k}} / \partial p_{k}\end{array}\right]$

$$
\begin{aligned}
& \lambda_{p}^{\prime}=(\partial \lambda / \partial p)^{\prime}=\left\{\partial \lambda / \partial p_{1}, \ldots, \partial \lambda / \partial p_{K}\right\} \\
& q_{C}^{\prime}=(\partial q / \partial C)^{\prime}=\left\{\partial q_{1} / \partial C, \ldots, \partial q_{K} / \partial C\right\}
\end{aligned}
$$

$\mathrm{y} \lambda_{c}=\partial \lambda / \partial C$

de modo que:
(1.7) $\left[\begin{array}{l}Q_{p} \\ -\lambda_{p}^{\prime}\end{array}\right.$
$\left.\begin{array}{r}q_{c} \\ -\lambda_{c}\end{array}\right]=\left[\begin{array}{c}U \\ p^{\prime}\end{array}\right.$

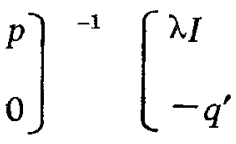


donde:

$$
\left[\begin{array}{ll}
U & p \\
p^{\prime} & 0
\end{array}\right]^{-1}=\left(p^{\prime} U^{-1} p\right)^{-1}\left[\begin{array}{l}
\left(p^{\prime} U^{-1} p\right) U^{-1} \\
\left(U^{-1} p\right)^{\prime}
\end{array}\right.
$$

dado, desde luego, que el hessiano y el hessiano orlado son matrices no singulares, entonces (1.7) y (1.8) implican:

$$
Q_{p}=\lambda U^{-1}-\left(p^{\prime} U^{-1} p\right)^{-1}\left\{\lambda\left(U^{-1} p\right)\left(U^{-1} p\right)^{\prime}+U^{-1} p q^{\prime}\right\}
$$

y

$$
q_{c}=\left(p^{\prime} U^{-1} p\right)^{-1}\left(U^{-1} p\right)
$$

\section{EL MODELO DE ASIGNACIÓN}

\subsection{Derivación}

Si las $K+1$ ecuaciones (1.1) y (1.3) tienen una solución apropiada, podemos obtener un sistema completo de ecuaciones de demanda, en el cual los precios y el ingreso son las variables determinantes del gasto. Esto se aplica también al modelo de asignación de gasto. Si fuera necesario o deseable, pueden introducirse otros factores tales como el tamaño y la composición del hogar o los acervos familiares.

El modelo de asignación:

$$
C_{k} / C=f_{k}\left(p_{k}, C\right) / \sum_{h=1}^{K} f_{h}\left(p_{h}, C\right) \text {, en donde } C_{k}=p_{k} q_{k}=\text { canti- }
$$

dad gastada en $k$, satisface idénticamente la condición de aditividad.

Las funciones $f_{k}$ se escogen de modo que las condiciones de Slutsky se cumplan para $k, l=1,2, \ldots, K$ :

$$
\partial q_{k} / \partial p_{l}+q_{l} \cdot \partial q_{k} / \partial C=\partial q_{l} / \partial p_{k}+q_{k} \cdot \partial q_{l} / \partial C
$$

En el Apéndice B.1 se demuestra que estas condiciones se satisfacen si:

$$
f_{k}\left(p_{k}, C\right)=F_{k}\left(p_{k} / C\right) \quad \text { para } k=1, \ldots, K
$$

es decir, si en las ecuaciones de demanda los precios y el ingreso aparecen solamente como cocientes precio/ingreso. Para especificar $F_{k}$, imponemos los siguientes requisitos prácticos y teóricos:

a) las condiciones de segundo orden del máximo de utilidad restringida deberán cumplirse.

b) Las ecuaciones de demanda deben ser las adecuadas para cualquier vector $\left(p^{\prime}, C\right)$ no negativo, inclusive en los valores extremos.

c) Las curvas de demanda deben ser continuas.

d) Los parámetros deben ser interpretables en términos económicos. 
e) Las funciones deben ser flexibles, de modo que puedan adaptarse a diversas situaciones de demanda.

f) Deben ser de la mayor simplicidad, compatible con los requisitos anteriores, y su tratamiento matemático.

Estas condiciones resultan en la especificación:

$$
f_{k}\left(p_{k}, C\right)=c_{k}\left(p_{k} / C\right)^{\alpha_{k}} \text { para } k=1, \ldots, K
$$

en las cuales $c_{k}$ y $\alpha_{k}$ pueden interpretarse como coeficientes de preferencia y parámetros de reacción, respectivamente.

La sustitución de (2.1.4) en (2.1.1) resulta en:

$$
\text { (2.1.5) } \quad q_{k}=c_{k}\left(p_{k} / C\right)^{\alpha_{k}-1} / \Sigma_{h} c_{h}\left(p_{h} / C\right)^{\alpha_{h}} \quad \text { para } k=1, \ldots, K
$$

Para garantizar el cumplimiento de las condiciones de segundo orden para el máximo es necesario y suficiente, según se demuestra en el Apéndice B2, que

(2.1.6) $\quad \alpha_{k}<1 \quad$ para $k=1, \ldots, K$

\subsection{Algunas propiedades}

De (2.1.5) se deduce que:

(2.2.1) $\quad E\left(q_{k}, C\right) \equiv \partial \log q_{k} / \partial \log C=1-\alpha_{k}+\sum_{h=1}^{K} w_{h} \alpha_{h}$

en el caso de las elasticidades "ingreso", y

(2.2.2) $\quad E\left(q_{k}, p_{l}\right)=-\left(1-\alpha_{l}\right) \delta_{k l}-w_{l} \alpha_{l}$

donde $\delta_{k l}=1$ si $k=l$, y $\delta_{k l}=0$ si $k \neq l$, para el caso de las elasticidades precio.

De (2.2.1) y (2.2.2) se derivan:

$$
\partial E\left(q_{k}, p_{l}\right) / \partial p_{l}=-C^{-1} \alpha_{l}^{2} q_{l}\left(1-w_{l}\right)<0
$$

$$
\partial E\left(q_{k}, C\right) / \partial C=-C^{-1} \sum_{h} w_{h}\left(\alpha_{h}-\bar{\alpha}\right)^{2}<0
$$

donde $\bar{\alpha} \equiv \sum_{h} w_{h} \alpha_{h}$

Esto significa que al aumentar $p_{t}$ y $C$, las elasticidades precio e ingreso disminuyen al mismo ritmo para todas las $k=1, \ldots, K$. La ecuación presupuesta (1.1) implica que:

a) Las funciones de demanda para $q_{1}, \ldots, q_{K}$, son homogéneos de grado 0 en $p$ y $C$. 

b) $\sum_{h} w_{h} E\left(q_{h}, C\right) \equiv 1$
c) $\sum_{h} w_{h} E\left(q_{h}, p_{k}\right) \equiv-w_{k}$

Una propiedad interesante es la forma de la curva de Engel cuando $C \rightarrow \infty$ :

$$
\begin{aligned}
\lim _{C \rightarrow \infty} q_{k} & \rightarrow \infty \text { si } \alpha_{k}<\min _{h}\left(\alpha_{h}+1\right) \\
& =\text { finito si } \alpha_{k}=\min _{h}\left(\alpha_{h}+1\right) \\
& =0 \text { si } \alpha_{k}>\min _{h}\left(\alpha_{h}+1\right)
\end{aligned}
$$

$\mathrm{Si}$ se conocen todas las elasticidades ingreso y cuando menos una elasticidad precio, todas las demás elasticidades precio pueden 'calcularse mediante (2.2.1) y (2.2.2).

El modelo de asignación del gasto puede deducirse de la función utilidad aditiva logarítmica: ${ }^{1}$

mediante

$$
\bar{u}\left(p^{\prime}, C\right)=\sum_{h}\left(c_{h} / \alpha_{h}\right)\left(p_{h} / C\right)^{\alpha_{h}}
$$

$$
q_{k}=\left(\partial \bar{u} / \partial p_{k}\right) /(\partial \bar{u} / \partial C)
$$

Por consiguiente, el modelo de asignación del gasto es llamado "modelo indirecto adilog".* El modelo de asignación puede ampliarse en varios sentidos, tomando igualmente en cuenta otros factores. Esto puede hacerse con mayor facilidad considerando los coeficientes de preferencia $c_{k}$ como funciones de tales variables adicionales; por ejemplo, la relación entre el consumo y, entre otras cosas, el tamaño de la familia a través de las $c_{k}$, ha sido examinada por Blokland y Somermeyer (1970).

3. MÉTodos de estimación de los PARÁMETROS DEL MODELO DE ASIGNACIÓN DEL GASTO

\subsection{Modelo de asignación con precios constantes (MAC)}

\subsubsection{Modelo aleatorio para los individuos}

Primero, se identifican las funciones de demanda con respecto a los individuos $i(=1, \ldots, I)$ a las cuales pertenecen, colocando subíndices $i$ a las variables y luego se hace aleatorio el modelo incorporando la perturbación aleatoria $u_{i h}(h=1, \ldots, K)$. De esto resulta que:

$$
C_{i k}=p_{i k} q_{i k}=c_{k}\left(p_{i k} / C_{i}\right) \alpha_{k} e u_{i k} /\left\{\sum_{h} c_{h}\left(p_{i k} / C_{i}\right) \alpha_{h} e u_{i h}\right\}
$$

1 Cf. Houthakker (1960).

* Se usará aquí la misma contracción adilog que en la versión inglesa. Otro tanto se hace más adelante en el caso de la expresión lognormal [T]. 
en donde $q_{i k}$ denota las cantidades de $k$ consumidas por el individero $i$, con gastos totales $C_{i}$, a precios $p_{i k}$, que resultan en gastos por rubro $C_{i k}$. Las ecuaciones (3.1.1.1) implican:

$$
\text { (3.1.1.2) } \quad \begin{aligned}
\log \left(C_{i k} / C_{i l}\right) & =\log \left\{c_{k} p_{i k} /\left(c_{l} p_{i l}\right)\right\} \\
& +\left(\alpha_{l}-\alpha_{k}\right) \log C_{i}+\left(u_{i k}-u_{i l}\right),
\end{aligned}
$$

para el caso de los cocientes por rubro de gasto, o en forma equivalente:

$$
\begin{aligned}
\log \left(q_{i k} / q_{i l}\right) & =\log \left\{c_{k} p_{i k}^{\alpha_{k}-1} / c_{l} p_{i l}-1\right. \\
& +\left(\alpha_{l}-\alpha_{k}\right) \log C_{i}+\left(u_{i k}-u_{i l}\right) .
\end{aligned}
$$

En ambos casos hay $\frac{1}{2} K(K-1)$ ecuaciones diferentes, aunque interrelacionadas para $k, l=1, \ldots, K$. Dado que los precios $p_{i k}$ son los mismos para todos los individuos, es decir:

$$
\text { (3.1.1.4) } \quad p_{i k} \equiv p_{k} \text {, }
$$

el primer término al lado derecho de (3.1.1.3) es una constante, diga$\operatorname{mos} \beta$.

Las estimaciones mínimo cuadráticas de $\left(\alpha_{k}-\alpha_{l}\right)$ en (3.1.1.2) que resultan de minimizar $\sum_{k} \sum_{i}\left(u_{i k}-u_{i l}\right)^{2}$, es decir,

$$
\left(\alpha_{k}-\alpha_{l}\right)^{*}=\sum_{i} \underline{\log }\left(C_{i k} / C_{i l}\right) \underline{\log } C_{i} / \sum_{i}\left(\underline{\log } C_{i}\right)^{2}
$$

son iguales a las de (3.1.3), a saber

$$
\left(\alpha_{k}-\alpha_{l}\right) *=\Sigma_{i} \underline{\log }\left(q_{i k} / q_{i l}\right) \underline{\log } C_{i} / \Sigma_{i}\left(\log C_{i}\right)^{2}
$$

en donde el subíndice (-) se añade a los logaritmos para indicar que estos últimos están medidos como desviaciones respecto de sus medias.

Estas estimaciones son insesgadas si $\operatorname{los} C_{1}$ son no aleatorios. Más aún, son mutuamente consistentes en el sentido de que:

$$
\left(\alpha_{k}-\alpha_{l}\right) *=-\left(\alpha_{l}-\alpha_{k}\right) * \text { (reversibilidad) }
$$

$\mathrm{y}$

(3.1.1.8) $\quad\left(\alpha_{k}-\alpha_{l}\right) *=\left(\alpha_{k}-\alpha_{j}\right) *-\left(\alpha_{l}-\alpha_{j}\right) *$ (triangularidad)

para $j, k, l=1, \ldots, K$;

y análogamente, en el caso de las estimaciones de doble asterisco basadas en (3.1.1.3). La ventaja práctica es que podemos confinar la esti-

- mación a un conjunto de diferencias $\alpha$, tomando una de las $\alpha$ (digamos la primera) como "base". Esto basta para calcular las elasticidades ingreso $E\left(q_{k}, C\right)$, mediante:

$$
E\left(q_{k}, C\right)=1+\sum_{h=1}^{K} w_{h}\left\{\left(\alpha_{h}-\alpha_{1}\right)-\left(\alpha_{h}-\alpha_{1}\right)^{*}-\left(\alpha_{k}-\alpha_{1}\right)\right\}^{*}
$$


Sin embargo, no siempre es posible aplicar el método de los mínimos cuadrados a los datos individuales, teóricamente debido a la incidencia de consumos cero en algunos rubros (que implican valores de menos infinito en los logaritmos), o prácticamente (si no se dispone de información individual) tiene que buscarse un método de estimación aplicable a datos agregados, tales como los recolectados en las encuestas de ingresos y gastos de hogares. La información obtenida de este modo es clasificada principalmente por grupos de ingresos, ya que el ingreso es el determinante más importante de los gastos en cada rubro. Por lo tanto concentraremos nuestra atención en este punto.

\subsubsection{Agregación por clases de ingreso}

De la suma de las ecuaciones (3.1.1.2) sobre los individuos que pertenecen a los grupos de ingreso $g(=1, \ldots, G)$ se obtiene:

$$
\begin{aligned}
& N_{\mathrm{g}}^{-1} \sum_{i \varepsilon\{g\}} \log \left(C_{i k} / C_{i l}\right) \\
& =N_{g}^{-1} \sum_{i \varepsilon\{g\}} \log \left\{c_{k} p_{i k}^{\alpha_{k}} /\left(c_{i} p_{i l}^{\alpha_{l}}\right)\right\} \\
& +\left(\alpha_{k}-\alpha_{l}\right) N_{g}^{-1} \sum_{i \varepsilon\{g\}}^{\Sigma} \log C_{i} \\
& +N_{g}^{-1} \sum_{i \varepsilon\{g\}} \log C_{i} \\
& +N_{g}^{-1} \sum_{i \varepsilon\{g\}}\left(u_{i k}-u_{i l}\right)
\end{aligned}
$$

de este modo, las relaciones quedan expresadas en términos de las medidas geométricas de las variables sobre los individuos dentro de cada clase de ingresos. Ya que en la práctica lo que se obtiene comúnmente son las medias aritméticas y no las geométricas, se intentará reemplazar las primeras por estas últimas.

$$
\begin{aligned}
\log \left(C_{g k} / C_{g l}\right) & =\log \left\{c_{k} p_{g k}^{\alpha_{k}} /\left(c_{l} p_{g l}^{\alpha_{l}}\right)\right\} \\
& +\left(\alpha_{k}-\alpha_{l}\right) \log C_{g}+\left(u_{g k}-u_{g l}\right)
\end{aligned}
$$

donde las variables que tienen el subíndice $g$ denotan las medias aritméticas de las variables correspondientes, a las cuales se han añadido los subíndices $i$, para las $i$ contenidas en $g$.

De igual modo, de (3.1.1.3) obtenemos:

$$
\begin{aligned}
\log \left(q_{g k} / q_{g l}\right) & =\log \left\{c_{k} p_{g k}^{\alpha_{k}-1} /\left(c_{l} p_{g l}^{\alpha_{1}-1}\right)\right\} \\
& +\left(\alpha_{k}-\alpha_{l}\right) \log C_{g}+\left(u_{g k}-u_{g l}\right)
\end{aligned}
$$

sin embargo, tales modificaciones se justifican solamente si las diferencias entre las medias de los logaritmos y los logaritmos de las medias 
(aritméticas) son iguales en todos los grupos de ingreso, o al menos cuando son más o menos pequeñas.

La aplicación de mínimos cuadrados a (3.1.2.2) y a (3.1.2.3) proporciona los estimadores

$$
\left(\alpha_{k}-\alpha_{l}\right)=\frac{\sum_{g=1}^{G} \log \left(C_{g k} / C_{g l}\right) \log C_{g}}{\sum_{g=1}^{G}\left(\log C_{g}\right)^{2}}
$$

y

$$
\left(\alpha_{k}-\alpha_{l}\right)=\frac{\sum_{g=1}^{G} \log \left(q_{g k} / q_{g l}\right) \log C_{g}}{\sum_{g=1}^{G}\left(\log C_{g}\right)^{2}}
$$

respectivamente, donde todas las variables en logaritmos están expresadas como desviaciones respecto de sus medias; formalmente, estas estimaciones son idénticas a las presentadas en (3.1.1.5) y (3.1.1.6), en donde simplemente se ha reemplazado $i$ por $g$.

3.1.3. El sesgo resultante de la aplicación del modelo de asignación con precios constantes a situaciones de precios variables

En la práctica, las categorías presupuestales distinguidas son heterogéneas en el sentido de que existen diferencias de precios entre sus componentes, las cuales indican diferencias de calidad. En vista de que puede variar la composición de estas categorías de acuerdo con el ingreso -en particular entre clases de éste- los precios medios pueden mostrar también cambios entre clases.

De acuerdo con Theil (1951), podemos suponer que entre el precio medio $p_{g k}$ del bien $k$ en la clase $g$ y el ingreso medio $C_{g}$ de esa clase existe la relación siguiente:

$$
\text { (3.1.3.1) } \quad \log p_{g k}=\gamma_{0 k}+\gamma_{1 k} \log C_{g}+\eta_{g k}
$$

en la cual $\eta_{g k}$ es un término de error, y $\gamma_{0 k}$ y $\gamma_{1 k}$ son constantes; $\gamma_{1 k}=$ $=E\left(p_{g k}, C_{g}\right)$ denota la "elasticidad cualitativa" de bienes compuestos con respecto al ingreso, supuesto independiente de éste.

Después de sustituir (3.1.3.1) en (3.1.2.2) y (3.1.2.3), los coeficientes de $\log C_{g}$ se convierten en:

$$
\beta_{1, k l}=\alpha_{l}\left(1-\gamma_{1 l}\right)-\alpha_{k}\left(1-\gamma_{1 k}\right)
$$

$$
\beta_{2, k l}=\beta_{1, k l}+\gamma_{1 l}-\gamma_{1 k},
$$

respectivamente. 
Por consiguiente, resulta claro que las estimaciones de $\left(\alpha_{k}-\alpha_{l}\right)$. de acuerdo con (3.1.2.4) y (3.1.2.5) son insesgadas si y solamente si

$\alpha_{k} \gamma_{1 l}=\alpha_{l} \gamma_{1 l}$ con respecto a $\beta_{1, k l}$

o bien $\left(1-\alpha_{k}\right) \gamma_{1 l}=\left(1-\alpha_{l}\right) \gamma_{l k}$ con respecto a $\beta_{2, k l}$

para $k, l=1, \ldots, K$; ambas estimaciones son insesgadas simultáneamente si y sólo si

$$
\gamma_{1 g} \equiv 0 \text { para } g=1, \ldots, G
$$

El impacto de estos sesgos sobre las estimaciones de las elasticidades ingreso puede derivarse de la manera siguiente:

$$
E\left(C_{k}, C\right)=1+\sum_{h=1}^{K} w_{h}\left(\alpha_{h}-\alpha_{k}\right)
$$

las cuales representan las elasticidades ingreso estimadas de acuerdo a (3.1.3.4).

Sus valores esperados (denotados por un superíndice + ) son:

$$
\begin{aligned}
E\left(C_{k}, C\right)^{+}= & 1+\sum_{h=1}^{K} w_{h}\left\{\alpha_{h}\left(1-\gamma_{1 h}\right)-\alpha_{k}\left(1-\gamma_{1 k}\right)\right\} \\
= & 1+\sum_{h=1}^{K} w_{h}\left(\alpha_{h}-\alpha_{k}\right)(=\text { al valor verdadero }) \\
& \quad-\sum_{h=1}^{K} w_{h}\left(\alpha_{h} \gamma_{1 h}-\alpha_{k} \gamma_{1 k}\right)(=\text { sesgo })
\end{aligned}
$$

Del mismo modo:

$$
E\left(q_{k}, C\right)^{*}=1+\sum_{h=1}^{K} w_{h}\left(\alpha_{h}-\alpha_{k}\right)
$$

la cual denota estimaciones de acuerdo a (3.1.2.5). Sus valores esperados son:

$$
\begin{aligned}
E\left(q_{k}, C\right)+= & +\sum_{h=1}^{K} w_{h}\left\{\alpha_{h}\left(1-\gamma_{1 h}\right)-\alpha_{k}\left(1-\gamma_{1 k}\right)+\gamma_{1 h}-\gamma_{1 k}\right\} \\
=1 & +\sum_{h=1}^{K} w_{h}\left(\alpha_{h}-\alpha_{k}\right) \quad(=\text { valor "verdadero") } \\
& +\sum_{h=1}^{K} w_{h}\left\{\left(1-\alpha_{h}\right) \gamma_{1 h}-\left(1-\alpha_{k}\right) \gamma_{1 k}\right\} \text { (= sesgo) }
\end{aligned}
$$

$E\left(C_{k}, C\right) *$ es un estimador insesgado de $E\left(C_{k}, C\right)$ si y solamente si

$$
\alpha_{k} \gamma_{1 k}=\sum_{h=1}^{\mathbf{K}} w_{h} \alpha_{h} \gamma_{1 h}
$$


$E\left(q_{k}, C\right)$ * es un estimador insesgado de $E\left(q_{k}, C\right)$ si y solamente si

$$
\left(1-\alpha_{k}\right) \gamma_{1 k}=\sum_{h=1}^{K} w_{h}\left(1-\alpha_{h}^{\prime}\right) \gamma_{1 h}
$$

Para todas las $k$ simultáneamente, estos últimos requisitos equivalen a las condiciones de insesgamiento de las estimaciones de las $\left(\alpha_{1}-\alpha_{k}\right)$ a saber, que $\alpha_{k} \gamma_{1 k}$ y $\left(1-\alpha_{k}\right) \gamma_{1 k}$ sean las mismas para todas las $k$; para alguna $k$ individual, las condiciones son más débiles en el sentido de que los productos $\alpha_{k} \gamma_{1 k}$ y $\left(1-\alpha_{k}\right) \gamma_{1 k}$ deben simplemente ser iguales a sus valores promedio para todos los rubros $h$, ponderados con las participaciones presupuestales $w_{h}$.

Si estas condiciones de insesgamiento no se satisfacen (al menos con un buen grado de aproximación), debe intentarse corregir el sesgo, mediante la estimación de éste. Esto requiere de la estimación de las $\gamma_{1 k}$ en (3.1.3.1); sin embargo, se requeriría además de la estimación de las $\alpha$ por separado en lugar de sus meras diferencias mutuas.

En vista de estas últimas exigencias podemos igualmente hacer uso de un modelo que tome en cuenta precios variables desde su formulación, que proporcione estimaciones de las $\alpha$ por separado. Esto puede hacerse como se indicará en la sección siguiente.

\section{3:2. Modelo de asignación con precios variables (MAV)}

Somermeyer, Hilhorst y Wit (1962) desarrollaron un método para estimar los parámetros del modelo de asignación del gasto mediante el análisis de series de tiempo. Para este fin (3.1.2.2) puede reescribirse:

$$
\begin{aligned}
\log C_{g k}-\log C_{g l} & =\alpha_{k} \log \left(p_{g k} / C_{g}\right) \\
& -\alpha_{l} \log \left(p_{g l} / C_{g}\right) \\
& +\left(u_{g k}-u_{g l}\right)
\end{aligned}
$$

para $k, l=1, \ldots, K$ y $g=1, \ldots, G$. Sumando sobre $l$, las $1 / 2 K(K-1)$ ecuaciones (3.2.1), en apariencia diferentes, se reducen al sistema de $K$ ecuaciones siguientes:

$$
\begin{aligned}
K \log C_{g k}-\sum_{l=1}^{K} \log C_{g l} & =K \alpha_{k} \log \left(p_{g k} / C_{g}\right) \\
& -\sum_{l=1}^{K} \alpha_{l} \log \left(p_{g l} / C_{g}\right) \\
& +\left(K u_{g k}-\sum_{l} u_{g l}\right)
\end{aligned}
$$

para $k=1, \ldots, K$.

En notación matricial:

$$
y=X \alpha+v,
$$




$$
\begin{aligned}
& \text { donde } y=\left[\begin{array}{c}
z_{\cdot 1} \\
\cdot \\
\cdot \\
z_{\cdot K}
\end{array}\right] \text { es un vector de } K G \text { elementos y } \\
& z^{\prime} \cdot k=\left\{-\sum_{l=1}^{K} \log \left(C_{1 l} / C_{l k}\right) \ldots-\sum_{l=1}^{K} \log \left(C_{G l} / C_{G k}\right)\right\}
\end{aligned}
$$

son vectores de $G$ elementos para $k=1, \ldots, K$; por otra parte,

$$
X=\left(\begin{array}{ccccc}
(K-1) \pi_{1} & -\pi_{2} & . & . & -\pi_{K} \\
-1 & (K-1)_{2} & . & . & \cdot \\
\cdot & \cdot & & \cdot \\
\cdot & \cdot & \cdot & \cdot \\
\cdot & \cdot & & \cdot \\
-\pi_{1} & -\pi_{2} & (K-1) \pi_{K}
\end{array}\right]
$$

es una matriz de orden $(K G) \times K$, $\pi_{k}^{\prime}=\left\{\log \left(p_{1 k} / C_{1}\right) \ldots \log \left(p_{G k} / C_{G}\right)\right\}$

son $K$ vectores de $G$ elementos; y $\alpha^{\prime}=\left\{\alpha_{1}, \ldots, \alpha_{K}\right\}$ es un vector de $K$ elementos. Por último,

$$
\begin{gathered}
v=\left[\begin{array}{c}
v_{1} \\
\cdot \\
\dot{v_{K}}
\end{array}\right] \text { es un vector de } K G \text { elementos, y } \\
v_{K}=\left\{\sum_{l=1}^{K}\left(u_{1 l}-u_{1 k}\right) \ldots \sum_{l=1}^{K}\left(u_{G l}-u_{G k}\right)\right\}
\end{gathered}
$$

El estimador mínimo cuadrático de $\alpha$ en (3.2.3), que minimiza $v^{\prime} v$ es:

$$
\hat{\alpha}=\left(X^{\prime} X\right)^{-1} X^{\prime} y
$$

\subsection{Varianzas de las estimaciones de punto}

La matriz de covarianzas $E\left(v v^{\prime}\right)$ de los errores $v$ en (3.2.3) es singular, de rango $(K-1) G$. Por lo tanto, pueden obtenerse estimaciones más eficientes de los parámetros mediante la aplicación del método de mínimos cuadrados generalizados, mediante una inversa generalizada de una estimación de $E\left(\nu v^{\prime}\right)$ que se inserta entre $x^{\prime}$ y $x$ y entre $X^{\prime}$ y $y$, lo cual equivale a una transformación lineal de las variables.

Sin embargo, los esfuerzos adicionales requeridos por tal refinamiento en el proceso de estimación no parecieron justificados, en particular dados 
los errores de medición inherentes tanto a las variables explicativas como a las explicadas; pueden además tenerse dudas acerca de la validez de los supuestos adoptados.

Por la misma razón, el vector de varianzas de las estimaciones de (3.2.4) de

$$
\operatorname{diag} E(\hat{\alpha}-\alpha) \widehat{(\alpha}-\alpha)^{\prime}=\operatorname{diag}\left[\left(X^{\prime} X\right)^{-1} X^{\prime} E\left(\nu v^{\prime}\right)\left(X^{\prime} X\right)^{-1}\right]
$$

ha sido obtenido en forma aproximada mediante:

$$
\text { est. } \operatorname{diag} E \hat{(\alpha-\alpha)} \widehat{(\alpha-\alpha)^{\prime}} \simeq \widehat{\sigma^{2}}\left(X^{\prime} X\right)^{-1}
$$

donde

$$
\widehat{\sigma^{2}}=\left(T-\hat{\Lambda) \hat{v^{\prime} v}} \text { y } \hat{v}=y\left[I-X\left(X^{\prime} X\right)^{-1} X\right]\right.
$$

si $T=$ número de observaciones y $\Lambda=$ número de parámetros que se busca estimar.

La fórmula anterior se aplica sin importar el método (MAC o MAV) adoptado para obtener estimaciones puntuales de los parámetros. No obstante, al juzgar el significado y el orden de magnitud de las estimaciones de los parámetros debe tenerse en mente que la naturaleza de (3.3.2) tiene el carácter de una mera aproximación.

Se han hecho supuestos adicionales para aproximar los errores estándar de las elasticidades ingreso (2.2.1), dependientes de las $\alpha_{k}$ así como de las $w_{h}$, a saber, los de covarianzas nulas entre las estimaciones de $\alpha$, y el supuesto de no aleatoreidad de los $w_{h}$. Evidentemente, estos supuestos son incorrectos, aunque los cálculos de las varianzas de las elasticidades pueden no verse afectados en forma importante ante desviaciones moderadas entre las hipótesis y la realidad. Mensink (1972) ha derivado fórmulas más exactas que toman en cuenta tanto las covarianzas de las estimaciones de $\alpha$ como la aleatoreidad de las $w_{h}$.

\section{Los datos y las estimaciones}

El modelo de asignación fue aplicado a los datos presentados en una encuesta de ingresos y gastos (1967). Esta investigación fue realizada en México durante 1963 y abarcó 5000 familias. Uno de los propósitos era el de hacer posible la estimación de funciones consumo e ingreso; los resultados habrian de usarse para predecir la demanda del consumidor en 1970 y 1975 , en particular la demanda de alimentos.

Los datos han sido agregados en nueve grupos de ingresos (véase el cuadro 2). Los datos han sido clasificados en seis grupos principales, a saber:

1. alimentos, bebidas y tabaco

2. vivienda, combustibles y electricidad

3. ropa y calzado

4. bienes durables

5. otros bienes y servicios

6. ahorro. 


\section{Cuadro 1}

Categorías de gasto

Grupos principales:

1. Alimentos $\mathrm{y}$ bebidas

2. Vivienda, combustibles, electricidad

3. Ropa y calzado

4. Bienes durables

5. Otros bienes y servicios

Subgrupos:

1.1. Maíz y sus productos

1.2. Trigo y sus productos

1.3. Arroz

1.4. Feculentas

1.5. Leguminosas

1.6. Azúcar

1.7. Verduras y legumbres

1.8. Frutas

1.9. Grasas y aceites

1.10. Leche y sus derivados

1.11. Carnes

1.12. Huevos

1.13. Pescado y mariscos

1.14. Bebidas

2.1. Renta

2.2. Combustibles y electricidad

2.3. Otros servicios

3.1. Ropa

3.2. Calzado

4.1. Muebles

4.2. Medios de transporte

4.3. Utensilios domésticos

5.1. Atención médica

5.2. Educación

5.3. Transporte

5.4. Recreación y cultura

5.5. Paseos y vacaciones

5.6. Otros gastos 
Cuadro 2

GRUPOS DE INGRESO

\begin{tabular}{lrrrr}
\hline 8 & & \multicolumn{3}{c}{ Pesos por mos } \\
\hline 1 & 0 & $\mathbf{a}$ & 300 \\
2 & 301 & $\mathbf{a}$ & 600 \\
3 & 601 & $\mathbf{a}$ & 1000 \\
4 & 1000 & $\mathbf{a}$ & 500 \\
5 & 1501 & $\mathbf{a}$ & 4500 \\
6 & 3001 & $\mathbf{a}$ & 6000 \\
7 & 4501 & $\mathbf{a}$ & 10000 \\
8 & 6001 & $\mathbf{a}$ & $\infty$ \\
\hline
\end{tabular}

El grupo 1 ha sido subdividido en 33 grupos, de los cuales hemos utilizado 14 en nuestras estimaciones, frente a los 33 manejados en la encuesta. Las clases 2 a 5 han sido subdivididas también en 33 grupos; los autores de la encuesta utilizaron sólo dos de éstos, y en nuestro trabajo adoptamos 14 para nuestras estimaciones. La clasificación utilizada aparece en el cuadro 1.

En la encuesta, la categoría 6. (es decir, el ahorro) ha sido tratado precisamente del mismo modo que los gastos propiamente dichos. Sin embargo, existen varias razones para omitir el ahorro de este tipo de análisis. Una razón práctica es que se acude a los logaritmos de las variables, de modo que los casos de desahorro pueden ocasionar pro-blemas. Una razón teórica es que las decisiones acerca de la asignación de los recursos vitales puede tomarse en dos etapas. En la primera, el ingreso es asignado entre consumo y ahorro (o desahorro), en el tiempo, en donde son importantes las consideraciones a largo plazo. En la segunda etapa, el monto de dinero señalado para el consumo por período es asignado entre las categorías de gasto; ésta es esencialmente una decisión a corto plazo.

En el estudio de la encuesta, se introdujeron las funciones siguientes:

1. Lineal: $C_{k g}=a_{k}+b_{k}\left(C_{g}+S a_{g}\right)+u_{k g}$

2. Bilogarítmica: $\log C_{k_{g}}=a+b \log \left(C_{g}+S a_{g}\right)+u_{k g}$

3. Semilogarítmica: $C_{k g}=a+b \log \left(C_{g}+S a_{g}\right)+u_{k g}$

4. Logarítmica inversa: $\log C_{k g}=a_{k}-b_{k} /\left(C_{g}+S a_{g}\right)+u_{s g}$

en donde $u_{s g}$ es un término de perturbación y $S a_{g}$ es el ahorro, en los rubros $k$ por grupo de ingreso $g$.

Las expresiones para las elasticidades ingreso son respectivamente:

$$
\begin{array}{rlrl}
\text { 1. } & E\left[C_{s},(C+S a)\right] & =(C+S a) /[(C+S a+a) / b] \\
& =b \\
\text { 3. } \quad, \quad " & =b /[a+b \log (C+S a)] \\
\text { 4. } \quad, \quad & =b /(C+S a)
\end{array}
$$


Esto corresponde a cuatro de las cinco funciones consumo comparadas entre sí por Prais y Hounthakker (1955).

El nivel promedio de ingreso y el gasto per capita medio en bienes separados, tomando en cuenta los grupos de ingreso, son respectivamente las variables exógenas y las endógenas en las funciones consumo. Esto presupone que las funciones son homogéneas de primer grado con respecto al tamaño de la familia, y que no importa la composición de la familia. Las limitaciones sobre los datos publicados hacen inevitable esta restricción.

En el estudio de la encuesta, las elasticidades han sido estimadas para todas las categorías de gasto, para las áreas menores de 2500 habitantes, para las mayores de 2600 y para todo el país. Los datos permiten una clasificación más en cinco tipos de áreas, caracterizadas según su número de habitantes.

\section{Cuadro 3}

Clasificación del lugar de residencia por tamaño de población

\begin{tabular}{|c|c|c|c|c|}
\hline & \multicolumn{4}{|c|}{ Numero de habitantes } \\
\hline $\begin{array}{l}1 \\
2 \\
3 \\
4 \\
5\end{array}$ & $\begin{array}{r}25 \\
100 \\
1500 \\
5000\end{array}$ & $\begin{array}{r}0 \\
501 \\
001 \\
000 \\
001\end{array}$ & $\begin{array}{l}a \\
a \\
a \\
a\end{array}$ & $\begin{array}{rr}2 & 500 \\
10 & 000 \\
150 & 000 \\
500 & 000\end{array}$ \\
\hline
\end{tabular}

En la investigación, cada una de las funciones consumo, de la 1 a la 4, han sido probadas en cuanto a la estimación de sus parámetros; se han utilizado además $q_{k}$ y $C_{k}$ como variables endógenas. Para cada categoría presupuestal, se escogió la función que dio las "mejores" estimaciones y en particular el mejor coeficiente de correlación. Evidentemente, este procedimiento resulta en funciones consumo que se ajustan mejor a aquellas formuladas con enfoques más apriorísticos, aunque carecen de base teórica, y en especial de consistencia.

Las estimaciones de las principales características aparecen resumidas en los cuadros 4 y 5 . De acuerdo con la encuesta (que de aquí en adelante denotaremos con ENC). Las estimaciones se obtienen con la función bilogarítmica de cuatro de los productos principales y con la función lineal de los bienes durables. Esto implica que, de las cinco elasticidades ingreso, cuatro permanecen constantes y una disminuye a medida que el ingreso aumenta.

Aplicando el modelo de asignación, aceptamos elasticidades ingreso decrecientes uniformemente (véase 2.2.4) en todas las categorias presupuestales; por ejemplo, la disminución es 0.058 si el ingreso per capita aumenta de 158 a 241 pesos por mes. Este último valor representa el ingreso promedio para el cual se calcularon las elasticidades.

Las diferencias obtenidas entre los resultados calculados según el MAC y la ENC, en las categorías 1 a 3 son muy considerables. Los resultados de la reestimación de ENC mediante mínimos cuadrados ponderados (que llamaremos $\mathrm{ENC}^{*}$ ) no difieren mucho de los del MAC. 
Cuadro 4

Coeficientes estimados de acuerdo a MAC, ENC y ENC* para los GRUPOS PRINCIPALES $Y$ LOS SUBGRUPOS NO ALIMENTICIOS, CON COEFICIENTES DE CORRELACIÓN

\begin{tabular}{|c|c|c|c|c|c|c|c|c|}
\hline \multirow{2}{*}{\multicolumn{2}{|c|}{$\begin{array}{c}\text { Categorla de } \\
\text { gas to }\end{array}$}} & \multicolumn{2}{|c|}{ MAC } & \multicolumn{3}{|c|}{ ENC } & \multicolumn{2}{|c|}{$\operatorname{suc}^{*}$} \\
\hline & & $\alpha_{1}-\alpha_{k}$ & $\mathbf{R}$ & Modelo & $b_{k}$ & $\mathbf{R}$ & $b_{k}$ & $\mathbf{R}$ \\
\hline \multicolumn{9}{|c|}{ Grapos principales } \\
\hline & 1 & & & doble-log & $\begin{array}{c}0.808 \\
(0.040)\end{array}$ & 0.991 & $\begin{array}{c}0.586 \\
(0.041)\end{array}$ & 0.999 \\
\hline & 2 & $\begin{array}{c}0.729 \\
(0.080)\end{array}$ & 0.955 & able-10g & $\begin{array}{c}1.200 \\
(0.045)\end{array}$ & 0.995 & $\begin{array}{c}1.145 \\
(0.035)\end{array}$ & 0.996 \\
\hline & 3 & $\begin{array}{c}0.516 \\
(0.041)\end{array}$ & 0.976 & doble-105 & $\begin{array}{c}0.691 \\
(0.049)\end{array}$ & 0.981 & $\begin{array}{c}1.035 \\
(0.040)\end{array}$ & 0.996 \\
\hline & 4 & $\begin{array}{c}1.431 \\
(0.082)\end{array}$ & 0.987 & lineal & $\begin{array}{c}0.132 \\
(0.012)\end{array}$ & 0.764 & $\begin{array}{c}0.111 \\
(0.013)\end{array}$ & 0.891 \\
\hline & 5 & $\begin{array}{c}1.023 \\
(0.039)\end{array}$ & 0.995 & doble-log & $\begin{array}{c}1.490 \\
(0.056)\end{array}$ & 0.995 & $\begin{array}{c}1.512 \\
(0.025)\end{array}$ & 0.999 \\
\hline
\end{tabular}

Szbegrupos no aldmenticios

\begin{tabular}{|c|c|c|c|c|c|}
\hline 2.2 & $\begin{array}{c}-0.600 \\
(0.021)\end{array}$ & 0.995 & & & \\
\hline 2.3 & $\begin{array}{c}0.168 \\
(0.072)\end{array}$ & 0.602 & & & \\
\hline 3.1 & & & doble-log & $\begin{array}{c}0.654 \\
(0.061)\end{array}$ & 0.967 \\
\hline 3.2 & $\begin{array}{c}-0.176 \\
(0.053)\end{array}$ & 0.705 & doble-log & $\begin{array}{c}0.440 \\
(0.073)\end{array}$ & 0.906 \\
\hline \multicolumn{6}{|l|}{4.1} \\
\hline 4.2 & $\begin{array}{c}0.809 \\
(0.124)\end{array}$ & 0.916 & & & \\
\hline $4 \cdot 3$ & $\begin{array}{c}0.078 \\
(0.113)\end{array}$ & & & & \\
\hline 5.2 & $\begin{array}{c}0.626 \\
(0.084)\end{array}$ & 0.934 & & & \\
\hline 5.3 & $\begin{array}{c}0.627 \\
(0.047)\end{array}$ & 0.378 & & & \\
\hline 5.4 & $\begin{array}{c}0.787 \\
(0.072)\end{array}$ & 0.967 & & & \\
\hline 5.5 & $\begin{array}{c}1.220 \\
(0.124)\end{array}$ & 0.960 & & & \\
\hline 5.6 & $\begin{array}{c}0.401 \\
(0.075)\end{array}$ & 0.881 & & & \\
\hline
\end{tabular}

Notación general para los cuadros 4 a 13.

MAC = Modelo de asignación con precios constantes con $q$ para las cantidades y $C$ para los gastos de consumo.

MAV = Modelo de asignación con precios variables.

ENC = Estimaciones de la encuesta.

ENC $^{*}=$ Reestimaciones de las ecuaciones de la encuesta.

Cifras entre paréntesis $=$ errores estándar estimados. 
Cuadro 5

Elasticidades INGReso ESTIMADAS DE ACUERDO A MAC, ENC Y ENC* PARA LOS PRINCIPALES GRUPOS Y SUBGRUPOS NO ALIMENTICIOS

\begin{tabular}{|c|c|c|c|c|}
\hline $\begin{array}{c}\text { Categoria de } \\
\text { gatito }\end{array}$ & $\begin{array}{l}c_{k} / c \\
(x)\end{array}$ & :AC & ENC & $\operatorname{ENC}^{5}$ \\
\hline \multicolumn{5}{|c|}{ Grupon principales } \\
\hline 1 & 50.8 & $\begin{array}{c}0.588 \\
(0.015)\end{array}$ & $\begin{array}{l}0.808 \\
(0.040)\end{array}$ & $\begin{array}{l}0.586 \\
(0.041)\end{array}$ \\
\hline 2 & 14.5 & $\begin{array}{l}1.316 \\
(0.048)\end{array}$ & $\begin{array}{l}1.200 \\
(0.045)\end{array}$ & $\begin{array}{c}1.145 \\
(0.035)\end{array}$ \\
\hline 3 & 13.1 & $\begin{array}{c}1.104 \\
(0.028)\end{array}$ & $\begin{array}{c}0.691 \\
(0.049)\end{array}$ & $\begin{array}{c}1.035 \\
(0.040)\end{array}$ \\
\hline 4 & 4.3 & $\begin{array}{c}2.018 \\
(0.047)\end{array}$ & 2.106 & 1.842 \\
\hline 5 & 17.3 & $\begin{array}{l}1.611 \\
(0.026)\end{array}$ & $\begin{array}{l}1.490 \\
(0.056)\end{array}$ & $\begin{array}{l}1.512 \\
(0.025)\end{array}$ \\
\hline \multicolumn{5}{|l|}{$\begin{array}{l}\text { Subgrupos no } \\
\text { alimentioios }\end{array}$} \\
\hline 2.1 & 10.9 & $\begin{array}{l}9.494 \\
(0.005)\end{array}$ & & \\
\hline 2.2 & 3.4 & $\begin{array}{c}0.705 \\
(0.017)\end{array}$ & . & \\
\hline 2.3 & 0.4 & $\begin{array}{l}1.716 \\
(0.056)\end{array}$ & & \\
\hline 3.1 & 0.7 & $\begin{array}{c}1.267 \\
(0.050)\end{array}$ & $\begin{array}{c}0.654 \\
(0.061)\end{array}$ & \\
\hline 3.2 & 3.6 & $\begin{array}{c}1.073 \\
(0.071)\end{array}$ & $\begin{array}{c}0.440 \\
(0.073)\end{array}$ & \\
\hline 4.1 & 0.9 & $\begin{array}{c}1.792 \\
(0.084)\end{array}$ & & \\
\hline 4.2 & 0.3 & $\begin{array}{l}3.425 \\
(0.126)\end{array}$ & & \\
\hline 4.3 & 3.2 & $\begin{array}{c}1.949 \\
(0.120)\end{array}$ & & \\
\hline 5.1 & 4.8 & $\begin{array}{c}0.907 \\
(0.027)\end{array}$ & & \\
\hline 5.2 & 2.4 & $\begin{array}{c}1.915 \\
(0.047)\end{array}$ & & \\
\hline 5.3 & 3.0 & $\begin{array}{l}1.917 \\
(0.035)\end{array}$ & & \\
\hline 5.4 & 1.3 & $\begin{array}{c}2.175 \\
(0.043)\end{array}$ & & \\
\hline 5.5 & 1.0 & $\begin{array}{c}2.872 \\
(0.063)\end{array}$ & & \\
\hline 5.6 & 4.8 & $\begin{array}{l}1.553 \\
(0.044) \\
\end{array}$ & & \\
\hline
\end{tabular}

Al comparar las elasticidades, debe tenerse en mente que de acuerdo con ENC y ENC*, el ingreso incluye el ahorro. Sin embargo dado que el ahorro constituye una proporción reducida del presupuesto (3\% en el cuarto grupo de ingresos), difícilmente cambia el panorama general.

La enorme participación del renglón de alimentos, las elasticidades comparativamente altas de alimentos calculadas al ingreso promedio $\mathrm{y}$ la pequeña participación presupuestal de los bienes durables, son características del bajo nivel de vida de México. 
Las perturbaciones estimadas mediante la aplicación del MAC no revelan heteroscedasticidad; esto puede deberse a la transformación logarítmica aplicada al modelo.

La ausencia de autocorrelación entre los residuos referentes a clases de ingreso sucesivas puede probarse mediante el coeficiente de Durbin y Watson:

$$
d=\sum_{g=2}^{G}\left(z_{g}-z_{g-1}\right)^{2} / \sum_{g=1}^{G} z_{g}^{2}
$$

Este se aplicó a las estimaciones MAC en las principales categorías de la 1 a la 5.

Para un caso como el presente, con sólo 9 observaciones, los intervalos de confianza no aparecen tabulados por Durbin y Watson (véase Koerts y Abrahamse, 1969); no obstante, la primera ecuación muestra autocorrelación positiva pero las otras no, a un nivel de significación del $5 \%$. No existe autocorrelación negativa.

Al comparar los resultados de MAC y ENC* en las categorías de gasto principales con respecto al coeficiente de correlación y los errores estándar, no existe razón alguna para preferir uno $\mathbf{u}$ otro modelo.

Las estimaciones de las categorías $1.1, \ldots, 1.14$ (todas las de alimentos) aparecen resumidas en los cuadros 6 y 7 . Los parámetros pertinentes del modelo de asignación fueron estimados directa e indirectamente, es decir, con el gasto total $(C)$ y el gasto en alimentos $\left(C_{1}\right)$ respectivamente, como variables explicativas. El método indirecto fue aplicado a la estimación:

a) mediante MAC para $C_{k}$ y $q_{k}$ (llamada MAC-C y MAC- $q$ respectivamente)

b) mediante MAV para $q_{k}, k=1.1, \ldots, 1.14$

El método directo se aplica a la estimación mediante el MAC, de nuevo tanto para $C_{k}$ como para $q_{k}, k=1.1, \ldots, 1.14$. Por consiguiente podemos calcular $E\left(C_{k}, C_{1}\right)$ y $E\left(q_{k}, C_{1}\right)$ de las estimaciones obtenidas por el método indirecto, a saber,

$$
\begin{aligned}
& E\left(C_{k}, C_{1}\right)=E\left(C_{k}, C\right) / E\left(C_{1}, C\right) \\
& E\left(q_{k}, C_{1}\right)=E\left(q_{k}, C\right) / E\left(C_{1}, C\right) ; k=1.1, \ldots, 1.14
\end{aligned}
$$

En la encuesta se presentan ecuaciones bilogarítmicas en cuatro casos, y lo mismo sucede con la logarítmica inversa; la semilogarítmica ocurre en tres casos y la forma lineal se da solamente una vez como función consumo preferida. Esto aparece bajo el encabezado ENC en el cuadro 6, el cual indica también si $C_{k} \circ q_{k}$ son las variables a ser explicadas.

A juzgar por los valores de los errores estándar y el coeficiente de correlación, la comparación de ENC con el MAC-C o el MAC- $q$ no favorece a ninguno. ${ }^{2}$ Los coeficientes de correlación para el MAC- $q$ no difie-

2 Una medida adecuada del comportamiento de los modelos de demanda es la "inexactitud de información" de las predicciones en un cierto período $t$ dada por 
CoEficientes Estimados de acuerdo al $M A C$ indirecto y al $M A C$ DiRecto con $q_{k}$ y $C_{k}$ como VARIABLES EXPLICADAS, RESPECTIVAMENTE, Y DE ACUERDO A ENC PARA LOS SUBGRUPOS ALIMENTICIOS

\begin{tabular}{|c|c|c|c|c|c|c|c|c|c|c|c|c|c|c|}
\hline \multirow{3}{*}{$\begin{array}{c}\text { Categoria } \\
\text { del } \\
\text { gasto }\end{array}$} & \multicolumn{6}{|c|}{ Indirot 00} & \multicolumn{8}{|c|}{$D \pm r \oplus 0 t 0$} \\
\hline & \multicolumn{2}{|l|}{$\mathrm{MAC}-\mathrm{q}$} & \multicolumn{2}{|l|}{ MAC-C } & \multicolumn{2}{|c|}{ MAV $-q$} & \multicolumn{2}{|l|}{ MAC-q } & \multicolumn{2}{|c|}{$\mathrm{MAC}-\mathrm{C}$} & \multicolumn{4}{|c|}{ ENC } \\
\hline & $\overline{\alpha_{1,1}-\alpha_{1 k}}$ & $\mathbf{R}$ & $\overline{\alpha_{1.1}-\alpha_{1 k}}$ & $\mathbf{R}$ & $\alpha_{1 k}$ & $\mathbf{R}$ & $1.1^{-\alpha, j}$ & $R$ & $\overline{\alpha_{1.1}-\bar{\alpha}_{1 \mathrm{k}}}$ & $R$ & Modelog & a & b & $\mathbf{R}$ \\
\hline 1.1 & & & & & 0.085 & 945 & & & & & semi-log & $\begin{array}{l}32.455 \\
(1.851)\end{array}$ & $\begin{array}{l}4.125 \\
(0.317)\end{array}$ & -0.980 \\
\hline 1.2 & $\begin{array}{c}1.711 \\
(0.186)\end{array}$ & 0.955 & $\begin{array}{c}1.198 \\
(0.127)\end{array}$ & 0.957 & 0.088 & & $\begin{array}{l}0.897 \\
(0.061)\end{array}$ & 0.982 & $\begin{array}{c}0.624 \\
(0.048)\end{array}$ & 0.977 & doble-log & $\begin{array}{r}-0.130 \\
(0.165)\end{array}$ & $\begin{array}{c}0.244 \\
(0.031)\end{array}$ & 0.945 \\
\hline 1.3 & $\begin{array}{c}1.592 \\
(0.115)\end{array}$ & 0.979 & $\begin{array}{c}1.238 \\
(0.136)\end{array}$ & 0.954 & 0.524 & & $\begin{array}{c}0.823 \\
(0.040)\end{array}$ & 0.991 & $\begin{array}{c}0.647 \\
(0.050)\end{array}$ & 0.977 & log-inverso & $\begin{array}{l}0.011 \\
(0.031)\end{array}$ & $\begin{array}{r}-89.064 \\
(5.711)\end{array}$ & 0.986 \\
\hline 1.4 & $\begin{array}{l}1.605 \\
(0.232)\end{array}$ & 0.924 & $\begin{array}{c}1.502 \\
(0.192)\end{array}$ & 0.940 & 0.438 & & $\begin{array}{c}0.867 \\
(0.057)\end{array}$ & 0.983 & $\begin{array}{c}0.800 \\
(0.051)\end{array}$ & 0.984 & $\log$-inverso & $(0.047)$ & $\begin{array}{r}-108.891 \\
(8.713)\end{array}$ & 0.978 \\
\hline 1.5 & $\begin{array}{c}0.473 \\
(0.127)\end{array}$ & 0.784 & $\begin{array}{l}0.610 \\
(0.146)\end{array}$ & 0.820 & 0.208 & & $\begin{array}{c}0.267 \\
(0.049)\end{array}$ & 0.883 & $\begin{array}{c}0.345 \\
(0.051)\end{array}$ & 0.922 & semi-log & $\begin{array}{c}5.309 \\
(0.134)\end{array}$ & $\begin{array}{l}-0.551 \\
(0.023)\end{array}$ & -0.994 \\
\hline 1.6 & $\begin{array}{l}1.281 \\
(0.173)\end{array}$ & 0.733 & $\begin{array}{c}0.990 \\
(0.156)\end{array}$ & 0.811 & 0.340 & & $\begin{array}{c}0.648 \\
(0.049)\end{array}$ & 0.980 & $\begin{array}{c}0.536 \\
(0.046)\end{array}$ & 0.971 & doble-log & $\begin{array}{c}0.008 \\
(0.038)\end{array}$ & $\begin{array}{c}0.221 \\
(0.007)\end{array}$ & 0.996 \\
\hline 1.7 & $\begin{array}{c}1.857 \\
(0.257)\end{array}$ & 0.930 & $\begin{array}{c}1.622 \\
(0.233)\end{array}$ & 0.925 & 0.236 & & $\begin{array}{c}1.000 \\
(0.059)\end{array}$ & 0.986 & $\begin{array}{c}0.877 \\
(0.053)\end{array}$ & 0.986 & doblo-log & $\begin{array}{l}0.162 \\
(0.157)\end{array}$ & $\begin{array}{c}0.322 \\
(0.029)\end{array}$ & 0.969 \\
\hline 1.8 & $\begin{array}{l}2.682 \\
(0.214)\end{array}$ & 0.975 & $\begin{array}{c}2.642 \\
(0.160)\end{array}$ & 0.986 & 0.200 & & $\begin{array}{c}1.393 \\
(0.064)\end{array}$ & 0.992 & $\begin{array}{l}1.358 \\
(0.069)\end{array}$ & 0.990 & lineal $3 /$ & $\begin{array}{c}0.684 \\
(0.327)\end{array}$ & $\begin{array}{c}0.009 \\
(0.001)\end{array}$ & 0.970 \\
\hline 1.9 & $\begin{array}{l}1.767 \\
(0.212)\end{array}$ & 0.946 & $\begin{array}{c}1.403 \\
(0.174)\end{array}$ & 0.943 & 0.180 & & $\begin{array}{l}0.340 \\
(0.052)\end{array}$ & 0.988 & $\begin{array}{r}0.748 \\
(0.041)\end{array}$ & 0.988 & semi-log $\frac{2}{2}$ & -11.087 & $\begin{array}{l}3.539 \\
(0.090)\end{array}$ & 0.998 \\
\hline 1.10 & $\begin{array}{l}2.305 \\
(0.198)\end{array}$ & 0.971 & $\begin{array}{l}1.730 \\
(0.144)\end{array}$ & 0.973 & 0.042 & & $\begin{array}{l}1.186 \\
(0.092)\end{array}$ & 0.977 & $\begin{array}{c}0.868 \\
(0.099)\end{array}$ & 0.950 & $\operatorname{cob} 2 \theta-10 \sigma^{8}$ & $\begin{array}{r}-0.110 \\
(0.259)\end{array}$ & $\begin{array}{c}0.455 \\
(0.048)\end{array}$ & 0.962 \\
\hline 1.11 & $\begin{array}{c}2.581 \\
(0.215)\end{array}$ & 0.973 & $\begin{array}{l}2.712 \\
(0.179)\end{array}$ & 0.983 & -0.320 & & $\begin{array}{c}1.343 \\
(0.062)\end{array}$ & 0.992 & $\begin{array}{c}1.397 \\
(0.073)\end{array}$ & 0.989 & log-invers of & $\begin{array}{l}4.478 \\
(0.036)\end{array}$ & $\begin{array}{r}-262.902 \\
(6.643)\end{array}$ & 0.998 \\
\hline 1.12 & $\begin{array}{l}2.314 \\
(0.182)\end{array}$ & 0.976 & $\begin{array}{l}1.993 \\
(0.200)\end{array}$ & 0.362 & 0.287 & & $\begin{array}{l}1.199 \\
(0.062)\end{array}$ & 0.989 & $\left(\begin{array}{l}1.039 \\
(0.071)\end{array}\right.$ & 0.982 & log-inverso & $\begin{array}{l}0.380 \\
(0.060)\end{array}$ & $\begin{array}{r}-153.109 \\
(11.221)\end{array}$ & 0.955 \\
\hline 1.13 & $\begin{array}{l}2.420 \\
(0.139)\end{array}$ & 0.987 & $\begin{array}{l}2.848 \\
(0.145)\end{array}$ & 0.990 & 0.747 & & $\begin{array}{l}1.229 \\
(0.093)\end{array}$ & 0.978 & $\begin{array}{c}1.446 \\
(0.104)\end{array}$ & 0.980 & $10 \mathrm{~B}$-inverso & $\begin{array}{l}1.939 \\
(0.113)\end{array}$ & $\begin{array}{r}-278.746 \\
(20.924)\end{array}$ & 0.981 \\
\hline 1.14 & $\begin{array}{c}1.317 \\
(0.212)\end{array}$ & 0.903 & $\begin{array}{c}1.629 \\
(0.185)\end{array}$ & 0.951 & 0.215 & & $\begin{array}{c}0.674 \\
(0.109)\end{array}$ & 0.908 & $\begin{array}{c}0.857 \\
(0.059)\end{array}$ & 0.982 & & & & \\
\hline
\end{tabular}

a La variable endógena es $C_{k}$ y en todos los demás casos $q_{k}$. 


\section{Cuadro 7}

ELASTICIDADES INGRESO ESTIMADAS DE ACUERDO AL $M A C$, AL $M A V$ INDIRECTO Y DE ACUERDO A ENC Y ENC $C^{*}$ PARA LOS GASTOS Y CANTIDADES CONSUMIDAS DE ALIMENTOS, RESPECTIVAMENTE

\begin{tabular}{|c|c|c|c|c|c|c|c|c|c|}
\hline \multirow{3}{*}{$\begin{array}{c}\text { Categoria } \\
\text { dol } \\
\text { gas to }\end{array}$} & \multirow{3}{*}{$\begin{array}{l}c_{k} / c \\
(\%)\end{array}$} & \multirow{2}{*}{\multicolumn{2}{|c|}{$\frac{E(C, 0)}{\text { MAC }}$}} & \multicolumn{3}{|c|}{$B(q, 0)$} & \multirow{2}{*}{\multicolumn{2}{|c|}{ Enc }} & \multirow{3}{*}{$\frac{\operatorname{Enc}^{k}}{E\left(C_{B}, C\right)^{2}}$} \\
\hline & & & & \multicolumn{2}{|c|}{ MAC } & \multirow{2}{*}{$\frac{\text { MAV }}{\text { Indireoto }}$} & & & \\
\hline & & Indireo to & Direoto & Indiraoto & Direoto & & $\overline{\mathrm{D}}\left(\mathrm{C}_{\mathrm{B}}, \mathrm{C}\right)$ & $E\left(q_{1}, c\right)$ & \\
\hline 1.1 & 5.0 & -0.390 & -0.279 & -0.473 & -0.402 & 0.576 & & -0.408 & -0.216 \\
\hline 1.2 & 5.3 & 0.306 & 0.345 & 0.533 & 0.493 & 0.574 & & 0.244 & 0.162 \\
\hline 1.3 & 1.0 & 0.329 & 0.367 & 0.463 & 0.420 & 0.318 & & 0.396 & 0.412 \\
\hline 1.4 & 1.0 & 0.485 & 0.520 & 0.470 & 0.464 & 0.368 & 0.484 & - & 0.484 \\
\hline 1.5 & 3.1 & -0.040 & 0.066 & -0.194 & -0.135 & 0.504 & - & -0.237 & 0.033 \\
\hline 1.6 & 1.5 & 0.183 & 0.256 & 0.280 & 0.281 & 0.426 & 0.229 & - & 0.221 \\
\hline 1.7 & 2.1 & 0.555 & 0.597 & 0.604 & 0.597 & 0.487 & 0.322 & - & 0.322 \\
\hline 1.8 & 2.5 & 1.155 & 1.077 & 1.104 & 0.989 & 0.509 & 0.737 & - & 0.737 \\
\hline 1.9 & 3.7 & 0.426 & 0.468 & 0.566 & 0.536 & 0.520 & 0.438 & - & 0.438 \\
\hline 1.10 & 6.2 & 0.619 & 0.588 & 0.882 & 0.781 & 0.602 & 0.455 & - & 0.455 \\
\hline 1.11 & 12.1 & 1.196 & 1.115 & 1.043 & .0 .939 & 0.814 & 1.168 & - & 9.168 \\
\hline 1.12 & 2.8 & 0.773 & 0.759 & 0.887 & 0.795 & 0.457 & - & 0.680 & 0.712 \\
\hline $1+13$ & 0.9 & 1.276 & 1.163 & 0.950 & 0.825 & 0.187 & - & 1.238 & 1.647 \\
\hline 1.14 & 3.5 & 0.559 & 0.557 & 0.301 & 0.271 & 0.500 & - & - & - \\
\hline
\end{tabular}


ren sistemáticamente de aquellos del MAC-c. El MAC directo muestra coeficientes de correlación mayores que el MAC indirecto.

Las diferencias entre las elasticidades calculadas mediante el MAV y el MAC- $q$ indirecto, respectivamente, son atribuibles a la diversidad en el método de estimación. Solamente en 7 de los 14 casos se tiene estimado el sesgo en MAV- $q$ de acuerdo con el signo correcto (3.1.1.6), según el cual puede darse explicación a las diferencias. Cuando el sesgo estimado es de 0.090 o más, el signo es correcto en cinco de cada seis casos. Solamente en el caso de la categoría 1.14 se encuentra un sesgo estimado de acuerdo con (3.1.3.7) - que explica más de la mitad de la diferencia.

\section{LA ESTRUCTURA DE PRECIOS Y LAS ELASTICIDADES CUALITATIVAS}

Las 14 categorías incorporadas dentro del gran grupo de alimentos, bebidas y tabaco constituyen agregados de bienes y por lo tanto son muy heterogéneas.

Un aumento en el consumo de un bien puede considerarse como la suma de dos componentes, a saber,

$$
\Delta C_{k}=p_{k} \Delta q_{k}+q_{k} \Delta p_{k},
$$

es decir, un cambio en el valor del consumo $\Delta C_{k}$ resulta de un cambio en el precio $\Delta p_{k}$, directa e indirectamente a través de su impacto sobre la cantidad $\Delta q_{k}$ consumida. ${ }^{3}$

La ecuación (5.1) implica que

$$
E\left(C_{k}, C\right)=E\left(q_{k}, C\right)+E\left(p_{k}, C\right)
$$

donde $E\left(p_{k}, C\right)$ denota la elasticidad cualitativa, suponiendo que las diferencias de calidad (por ejemplo, si se sustituye mantequilla por margarina, el precio promedio de la categoría de grasas aumenta). Debe notarse que si se conocen dos de las elasticidades de (5.2), la tercera puede calcularse.

Por lo general, las diferencias regionales de precios deben hacerse a un lado en el cálculo de las elasticidades cualitativas, debido a que estas diferencias son por lo común independientes de la calidad de los bienes y se encuentran indirectamente ante la opción del consumidor (al cambiar éste su lugar de residencia).

Las diferencias regionales en el caso de la producción y de la distribución, en especial los costos de transporte, pueden afectar el precio promedio de un bien según el grupo de ingreso. Más aún, según se observa en el cuadro 11, las familias mayores tienden a tener ingresos mayores.

Sin embargo, el ingreso y el tamaño del hogar guardan una correlación positiva con la edad del jefe.

$I_{t}=\Sigma w_{s t} \log \left(w_{s t} / \widehat{w_{s t}}\right)$, es decir, el cambio de información esperado que obtenemos al sustituir $w_{i t}$ por $\widehat{w}_{i t}$ (véase Theil y Mnookin, 1966).

3 Véase Theil, 1955. 
De acuerdo con el modelo de asignación, esperamos que el precio medio aumente con el ingreso, y que disminuya a medida que la familia crezca (véase el apéndice $C$ ). Por lo tanto, el tamaño de la familia y el ingreso tienen efectos opuestos sobre los precios medios por grupo de ingreso. En consecuencia, las diferencias de precios de los rubros, entre clases de ingreso, reflejan en parte diferencias interregionales en la distribución de los hogares según el ingreso y el tamaño de la familia.

En el cuadro 8 se resumen los precios medios por grupo de ingreso en todas las categorías de alimentos. Los rubros presupuestales $1.1,1.5,1.8,1.11,1.13$ y 1.14 muestran los precios medios que crecen en forma casi sostenida con el ingreso. Por añadidura, existe una tendencia en los rubros 1.3 y 1.6 a crecer y en los rubros 1.2 y 1.10 a decrecer.

\section{Cuadro 8}

Precios PRomedio de ALIMENTOS EN PESOS POR KILOGRAMO, POR GRUPOS DE INGRESO

\begin{tabular}{|c|c|c|c|c|c|c|c|c|c|}
\hline \multirow[t]{2}{*}{ Rubro } & \multicolumn{9}{|c|}{ Grupos d $1 \mathrm{ng} \mathrm{g}$ eso } \\
\hline & 1 & 2 & 3 & 4 & 5 & 6 & 7 & 8 & 9 \\
\hline $\begin{array}{l}1.1 \\
1.2 \\
1.3 \\
1.4 \\
1.5 \\
1.6 \\
1.7 \\
1.8 \\
1.9 \\
1.10 \\
1.11 \\
1.12 \\
1.13 \\
1.14\end{array}$ & $\begin{array}{l}1.04 \\
3.42 \\
3.24 \\
1.29 \\
2.57 \\
1.84 \\
1.88 \\
1.43 \\
7.36 \\
1.38 \\
8.34 \\
8.33 \\
6.88 \\
4.65\end{array}$ & $\begin{array}{l}1.04 \\
3.22 \\
3.28 \\
1.29 \\
2.52 \\
1.70 \\
1.98 \\
1.34 \\
7.42 \\
1.33 \\
9.78 \\
7.03 \\
5.30 \\
6.86\end{array}$ & $\begin{array}{r}1.12 \\
3.18 \\
3.23 \\
1.59 \\
2.81 \\
1.77 \\
1.97 \\
1.55 \\
7.38 \\
1.37 \\
10.66 \\
8.58 \\
6.33 \\
6.60\end{array}$ & $\begin{array}{r}1.28 \\
3.04 \\
3.12 \\
1.68 \\
3.12 \\
1.75 \\
2.07 \\
1.77 \\
7.45 \\
1.37 \\
12.57 \\
8.32 \\
10.37 \\
6.70\end{array}$ & $\begin{array}{r}1.32 \\
3.10 \\
3.14 \\
1.66 \\
3.30 \\
1.77 \\
2.12 \\
1.85 \\
7.09 \\
1.40 \\
13.18 \\
8.41 \\
9.60 \\
6.35\end{array}$ & $\begin{array}{r}1.39 \\
3.24 \\
3.02 \\
1.71 \\
3.67 \\
1.80 \\
2.08 \\
1.86 \\
7.43 \\
1.19 \\
14.48 \\
7.13 \\
11.96 \\
7.65\end{array}$ & $\begin{array}{r}1.42 \\
2.88 \\
3.13 \\
1.72 \\
4.06 \\
1.94 \\
2.03 \\
2.01 \\
7.19 \\
1.15 \\
14.46 \\
7.92 \\
12.12 \\
10.63\end{array}$ & $\begin{array}{r}1.41 \\
2.47 \\
3.44 \\
1.70 \\
4.10 \\
1.95 \\
2.30 \\
1.96 \\
7.59 \\
1.12 \\
14.72 \\
8.05 \\
13.18 \\
14.08\end{array}$ & $\begin{array}{r}1.66 \\
2.79 \\
3.19 \\
1.69 \\
4.73 \\
1.77 \\
2.09 \\
1.77 \\
6.79 \\
0.89 \\
14.88 \\
8.85 \\
14.94 \\
11.05\end{array}$ \\
\hline
\end{tabular}

En el cuadro 9 se presentan los precios promedio de algunos bienes alimenticios seleccionados, distinguidos según el tamaño del lugar de residencia y en varios grupos de ingreso. Tres de estos rubros se discutirán brevemente.

a) Maíz. Los renglones de 9 muestran precios promedio que generalmente aumentan al elevarse el ingreso. Las columnas de 9 muestran que los precios por lo general aumentan cuando crece el tamaño de la población de residencia. Estas tendencias no son, sin embargo, independientes entre sí, ya que hablando en términos relativos, las clases de ingresos superiores son más frecuentes en los pueblos que en las aldeas;

b) Legumbres. Las diferencias por grado de urbanización no son sistemáticas (véase el cuadro 9). Los renglones de 9 muestran que los precios se elevan al aumentar el ingreso. El cuadro 8 muestra el mismo fenómeno para todo el país;

c) Aceites y grasas. En este renglón las diferencias entre lugares de distintos tamaños no son tampoco sistemáticas. Los renglones del cuadro 9 muestran variaciones irregulares. 
Cuadro 9

Precios PROMEdIO REgIONALES PARA GRUPOS DE INGRESO Y RUBROS DE ALIMENTOS SELECCIONADOS

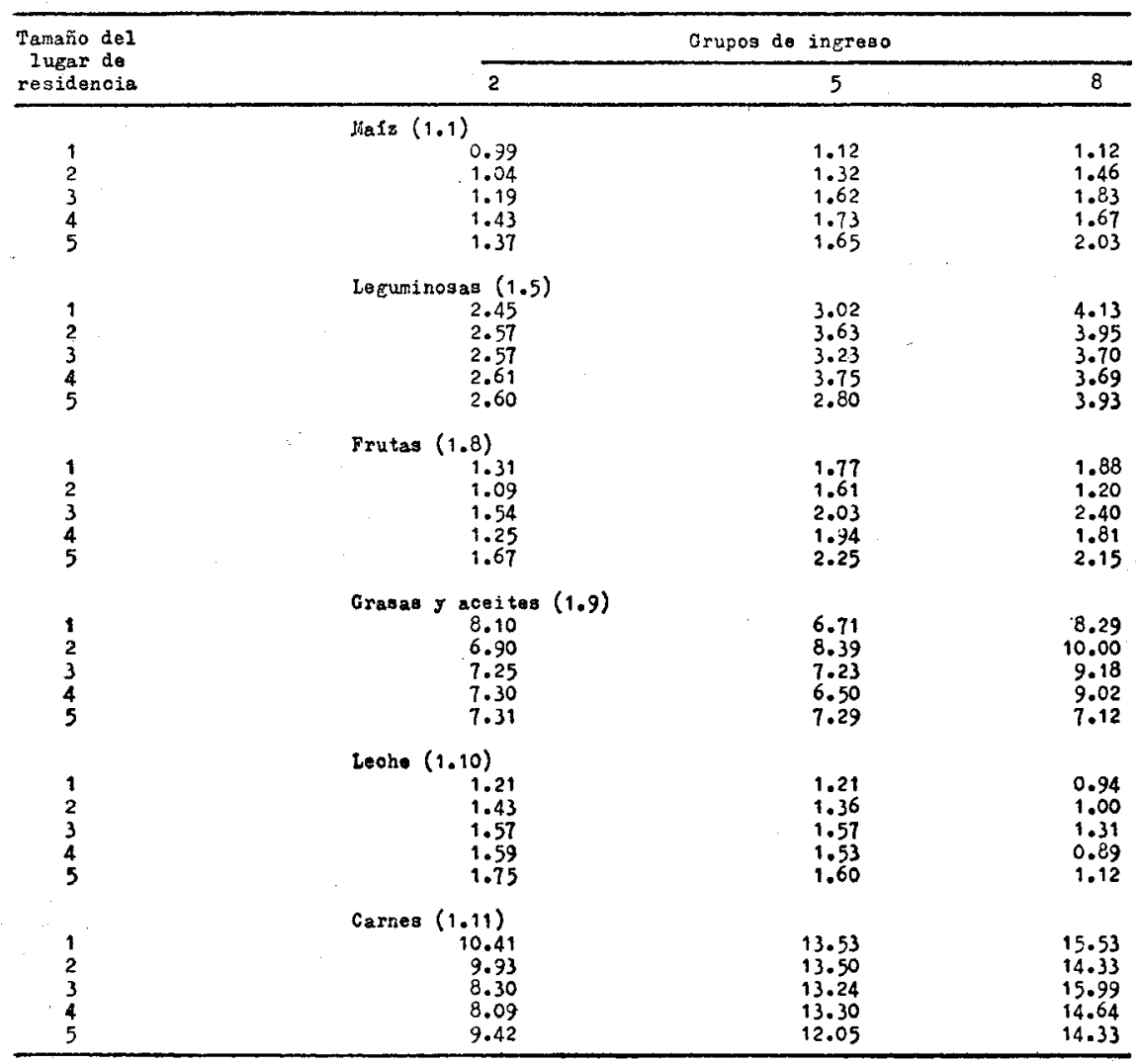

Cuadro 10

POBLACIÓN POR TAMAÑo DEL LUGAR DE RESIDENCIA (Porcientos)

\begin{tabular}{|c|c|c|c|c|c|c|}
\hline \multirow{2}{*}{$\begin{array}{l}\text { Grupos de } \\
\text { ingreso }\end{array}$} & \multicolumn{5}{|c|}{ Tameño del lugar de residencia } & \multirow{2}{*}{$\begin{array}{l}\text { Total } \\
\text { del } \\
\text { Pals }\end{array}$} \\
\hline & 1 & 2 & 3 & 4 & 5 & \\
\hline $\begin{array}{c}1 \\
2 \\
3 \\
4 \\
5 \\
6 \\
7 \\
8 \\
9 \\
\text { Total }\end{array}$ & $\begin{array}{r}25.6 \\
31.6 \\
23.4 \\
5.9 \\
9.6 \\
2.7 \\
0.7 \\
0.3 \\
0.1 \\
100.0\end{array}$ & $\begin{array}{r}16.5 \\
26.5 \\
24.7 \\
10.8 \\
13.9 \\
6.3 \\
0.7 \\
0.3 \\
-.0 \\
100.0\end{array}$ & $\begin{array}{r}7.6 \\
19.8 \\
18.4 \\
17.3 \\
25.3 \\
7.2 \\
3.1 \\
0.9 \\
0.4 \\
100.0\end{array}$ & $\begin{array}{r}4.1 \\
17.7 \\
21.8 \\
15.1 \\
20.7 \\
10.5 \\
3.9 \\
3.9 \\
2.2 \\
100.0\end{array}$ & $\begin{array}{r}1.3 \\
8.9 \\
18.9 \\
16.7 \\
23.9 \\
10.7 \\
7.7 \\
6.2 \\
6.8 \\
100.0\end{array}$ & $\begin{array}{r}16.0 \\
24.1 \\
21.9 \\
11.2 \\
16.4 \\
5.6 \\
2.2 \\
1.7 \\
1.1 . \\
100.0\end{array}$ \\
\hline
\end{tabular}




\section{Cuadro 11}

\section{TAMAÑO PROMEDIO DE HOGARES POR GRUPOS DE INGRESO $Y$ TAMAÑO DEL LUGAR DE RESIDENCIA}

\begin{tabular}{ccccccc}
\hline $\begin{array}{c}\text { Grupos de } \\
\text { ingsoso }\end{array}$ & \multicolumn{5}{c}{ Tamaño del lugar de residencia } & \multicolumn{1}{c}{$\begin{array}{c}\text { Total } \\
\text { del }\end{array}$} \\
\cline { 2 - 7 } & 1 & 2 & 3 & 4 & 5 & PaIs \\
\hline 1 & 5.2 & 4.6 & 4.7 & 3.4 & 3.7 & 5.2 \\
2 & 5.7 & 5.1 & 5.7 & 5.2 & 4.6 & 5.6 \\
3 & 6.0 & 6.0 & 5.5 & 6.0 & 5.4 & 5.5 \\
4 & 6.1 & 6.3 & 5.5 & 5.7 & 6.1 & 5.9 \\
5 & 7.1 & 6.1 & 6.0 & 6.0 & 5.8 & 6.3 \\
6 & 8.8 & 6.2 & 7.5 & 6.5 & 6.4 & 7.1 \\
7 & 6.7 & 8.0 & 7.8 & 6.3 & 6.3 & 6.6 \\
8 & 9.5 & 3.1 & 5.7 & 6.9 & 6.9 & 6.1 \\
9 & 8.0 & - & 7.5 & 8.7 & 7.9 & 7.3 \\
\hline
\end{tabular}

Cuadro 12

Elasticidades cualitativas de RUbros de alimentos Calculadas DE ACUERDO CON LOS MÉTODOS I A IV a

\begin{tabular}{|c|c|c|c|c|}
\hline \multirow{2}{*}{$\begin{array}{l}\text { Rubro de } \\
\text { al imentos }\end{array}$} & \multicolumn{4}{|c|}{ Wétodo de estimación } \\
\hline & $I$ & II & IIII & $\mathbf{I V}$ \\
\hline $\begin{array}{l}1.1 \\
1.2 \\
1.3 \\
1.4 \\
1.5 \\
1.6 \\
1.7 \\
1.8 \\
1.9 \\
1.10 \\
1.11 \\
1.12 \\
1.13 \\
1.14\end{array}$ & $\begin{array}{r}0.192 \\
-0.082 \\
0.016 \\
0.125 \\
0.270 \\
0.044 \\
0.069 \\
0.157 \\
0.000 \\
-0.126 \\
0.246 \\
0.032 \\
0.409 \\
0.375\end{array}$ & $\begin{array}{r}0.123 \\
-0.148 \\
-0.053 \\
0.056 \\
0.201 \\
-0.025 \\
0.000 \\
0.088 \\
-0.068 \\
-0.193 \\
0.176 \\
-0.036 \\
0.340 \\
0.306\end{array}$ & $\begin{array}{r}0.083 \\
-0.227 \\
-0.134 \\
0.015 \\
0.154 \\
-0.097 \\
0.049 \\
0.051 \\
-0.140 \\
-0.263 \\
0.153 \\
-0.114 \\
0.326 \\
0.258\end{array}$ & $\begin{array}{r}0.000 \\
-0.150 \\
0.290\end{array}$ \\
\hline
\end{tabular}

2 I Considerando el sesgo en la estimación directa de $E\left(C_{k}, C\right)$ y $E\left(q_{k}, C\right)$

II De las estimaciones directas de acuerdo a $M A C$

III De las estimaciones indirectas de acuerdo a $M A C$

IV De las estimaciones directas de acuerdo con el cuadro 13

En las columnas II y III del cuadro 12, se resumen dos series de elasticidades cualitativas para las categorias $1.1, \ldots, 1.14$; dichas series han sido calculadas mediante (5.2) a partir de las estimaciones MAC indirectas, de $E\left(C_{s}, C\right)$ y $E\left(q_{s}, C\right)$.

De acuerdo con (3.1.3.5) y (3.1.3.7):

$$
E\left(C_{k}, C\right)-E\left(q_{k}, C\right)=\gamma_{1 k}-\sum_{h=1}^{R} w_{h} \gamma_{1 h}
$$

Ya que en general $\Upsilon_{1 h}>0$, las elasticidades cualitativas basadas en (5.4) podrían estar subestimadas en relación con su valor "verdadero" (véase 3.1.3.1). 
De acuerdo con (3.1.3.1) a (3.1.3.3):

(5.4) $\quad \beta_{1, k l}-\beta_{2, k l}=\gamma_{1 k}-\gamma_{1 l}=E\left(p_{k}, C\right)-E\left(P_{l}, C\right)$ para $k, l=1, \ldots, K$.

Mediante (5.4) y las estimaciones MAC directas, se han calculado las $E\left(p_{k}, C\right)$ para $k=1.1, \ldots, 1.14$, suponiendo que $E\left(p_{1.9}, C\right)=0.4$

La columna I del cuadro 12 muestra valores de $E\left(p_{s}, C\right)$ mayores a los de las columnas II y III; esto confirma (5.3). La diferencia entre las cifras correspondientes en las columnas I y III es mayor, debido a que en (5.3) $w_{h}$ es igual a $C_{k} / C_{1} \circ$ a $C_{k} / C$ según se haya aplicado el MAC indirecto o el directo, respectivamente.

Un tercer método para calcular las elasticidades cualitativas es la estimación directa. Esto es posible si se conocen tanto las cantidades como los valores del consumo por grupo de ingreso. Para neutralizar diferencias en el tamaño de la familia y en la distribución del ingreso, de acuerdo con el tamaño del lugar de residencia, los precios promedio se han hecho iguales a las medias no ponderadas de los precios por clase de tamaño de los lugares de residencia.

Esta serie de precios se utilizó en cuatro categorías que cubren alrededor del $30 \%$ del gasto en alimentos, de modo de estimar las elasticidades cualitativas mediante (3.1.1.1):

$$
p_{k g}=\varepsilon_{k 0}+\varepsilon_{k 1} \log C_{g}+\nu_{k g}
$$

donde $\varepsilon_{k 0}$ y $\varepsilon_{k 1}$ son constantes y $\nu_{k g}$ son perturbaciones, lo cual implica que $E\left(p_{k}, C\right)=\varepsilon_{k 1} / p_{k}$.

Los resultados aparecen en el cuadro 13; éstos se utilizaron para calcular las elasticidades del cuadro 12, columna IV.

Para el caso de la categoría 1.1, existe cierta evidencia en favor del modelo (3.1.3.1), mientras que para las categorías 1.10 y 1.11 puede ser preferible el modelo (5.5), con elasticidades decrecientes. Las elasticidades en 1.1 y 1.10 tienen sesgos hacia arriba debido a las diferencias en los tamaños del lugar de residencia, según puede verse en el cuadro 9. Estos resultados concuerdan con las diferencias entre las columnas I y IV del cuadro 12.

Lo contrario se aplica a las categorías 1.11 , según el cuadro 9 .

Cuadro 13

Estimaciones directas DE $E\left(p_{s}, C\right)$ PARA RUBRos DE ALIMENTOS SELECCIONADOS

\begin{tabular}{|c|c|c|c|c|c|c|}
\hline \multirow{2}{*}{ Rubro } & \multicolumn{3}{|c|}{$p_{g k}-\varepsilon_{o k}+\xi_{1 k}{ }^{\log c} g$} & \multicolumn{3}{|c|}{$\log _{g k}=Y_{o k}{ }^{+}{ }_{1 k^{10 g}}{ }_{g}^{C}$} \\
\hline & $\mathrm{E}_{\mathrm{ok}}$ & $E_{1 k}$ & $\boldsymbol{R}$ & $Y_{\text {ok }}$ & $r_{1 k}$ & R \\
\hline 1.1 & $\begin{array}{l}-0.052 \\
(0.278)\end{array}$ & $\begin{array}{c}0.264 \\
(0.048)\end{array}$ & 0.886 & $\begin{array}{c}0.669 \\
(0.168)\end{array}$ & $\begin{array}{c}0.181 \\
(0.029)\end{array}$ & 0.909 \\
\hline 1.9 & $\left(\begin{array}{l}5.891 \\
1.062)\end{array}\right.$ & $\begin{array}{c}0.295 \\
(0.184)\end{array}$ & 0.405 & $\left\{\begin{array}{l}1.813 \\
0.135\end{array}\right\}$ & $\begin{array}{c}0.036 \\
(0.023)\end{array}$ & 0.399 \\
\hline 1.10 & $\begin{array}{c}2.511 \\
(0.293)\end{array}$ & $\begin{array}{c}-0.212 \\
(0.051)\end{array}$ & 0.820 & $\begin{array}{c}1.256 \\
(0.261)\end{array}$ & $\begin{array}{c}-0.176 \\
(0.045)\end{array}$ & 0.799 \\
\hline 1.11 & $\begin{array}{l}-6.389 \\
(2.405)\end{array}$ & $\begin{array}{c}3.285 \\
(0.416)\end{array}$ & 0.941 & $\begin{array}{c}0.810 \\
(0.287)\end{array}$ & $\begin{array}{c}0.293 \\
(0.050)\end{array}$ & 0.900 \\
\hline
\end{tabular}

4 Debido a la naturaleza homogénea del bien 1.9 (azúcar). 


\section{APENDICE A}

Problemas de estimación

\section{A.1. Agrupación óptima de las observaciones}

En el análisis de presupuestos familiares es usual resumir el gran número de observaciones recolectadas en medias de grupos con lo que se pierde información.

De aquí surgen las preguntas siguientes:

1) ¿Cuál es el impacto sobre el método y los resultados de estimación de los parámetros y sobre los resultados obtenidos que resultan de hacer agrupaciones?

2) ¿Cómo puede minimizarse la pérdida de información, o cómo puede maximizarse la eficiencia de las estimaciones una vez que se han hecho agrupaciones, si el número de grupos es fijo?

Supóngase que tenemos $I$ observaciones $\left(x_{g i}, y_{g i}\right)$, donde

$x_{g i}=$ valores de $x$ para el individuo $i$ en el grupo $g$

$y_{g i}=$ valor de $y$ para el individuo $i$ en el grupo $g$

para $g=1, \ldots, G ; i=1, \ldots, I_{g}$ y $\underset{g}{\Sigma} I=I$.

Suponemos que existe un modelo con las observaciones originales:

$$
\text { (A.1.1.) } \quad y_{g i}=\alpha+\beta x_{g i}+u_{g i}
$$

donde $u_{g i} \cong N\left(0, \sigma^{2}\right)$

El modelo después de agrupar es:

(A.1.2) $\quad \bar{y}_{g}=\alpha+\beta \bar{x}_{g}+\bar{u}_{g}$

donde $\bar{y}_{\mathrm{g}}=I_{g}^{-1} \sum_{i=1}^{g} y_{g i}$ y $\bar{x}_{g}$ y $\bar{u}_{g}$ tienen significados análogos a los anteriores.

Prais y Aitchison (1955) demostraron que:

a) después de agrupar, las estimaciones mínimo cuadráticas de $\alpha$ y $\beta$ son insesgadas;

b) las varianzas de las estimaciones son mayores después de agrupar;

c) esta pérdida de eficiencia depende de la forma de agrupar, y es mínima si sé combinan las observaciones más homogéneas en la medida de lo posible, con respecto a la variable explicativa principal. Específicamente, la suma de las varianzas intragrupos debe ser mínima (dado un número fijo de grupos). 
La pérdida de eficiencia al agrupar, aplicada a la regresión lineal con una sola variable explicativa es: 1

$$
\operatorname{var}(\bar{b}) / \operatorname{var}(b)=S\left(x^{2}\right) / S\left(\bar{x}^{2}\right)=S\left(x^{2}\right) /\left(S\left(x^{2}\right)-S\left(x^{\prime 2}\right)\right)>1,
$$

donde $b$ es la estimación mínimo cuadrática del coeficiente de regresión $\beta$ de las observaciones originales y. $\bar{b}$ es la estimación mínimo cuadrática de $\beta$ con las observaciones agrupadas. Asimismo:

$$
\begin{aligned}
& S\left(x^{2}\right)=\sum_{g} \sum_{i}\left(x_{g i}-\overline{\bar{x}}\right)^{2}, \text { donde } \overline{\bar{x}}=I^{-1} \sum_{g} \sum_{i \varepsilon\{g\}} x_{g i} ; \\
& S\left(\overline{x^{2}}\right)=\sum_{g} I_{g}\left(\bar{x}_{g}-\overline{\bar{x}}\right)^{2} \text {, es decir, la suma de cuadrados entre grupos; } \\
& S^{\prime}\left(x^{2}\right)=\sum_{g} \sum_{i \varepsilon\{g\}}\left(x_{g i}-\bar{x}_{g}\right)^{2} \text {, es decir, la suma de cuadrados intra grupos. }
\end{aligned}
$$

La pérdida de eficiencia depende solamente de la distribución de $x$ y del método de agrupación. La eficiencia de $\bar{b}$ se aproxima a la de $b$ a medida que $S\left(x^{2}\right)$ disminuye (en sentido relativo). Cramer (1964) calculó la pérdida de eficiencia en un caso especial, suponiendo que

a) $x_{g i}=\log z_{g i}$ donde $z_{g i}$ es el ingreso de la familia $g_{i}$,

b) $x_{g i}$ es una muestra tomada de una población con distribución normal, de modo que $Z$ tiene una distribución lognormal,

c) el método de agrupación divide el intervalo de $x_{g i}$ en $G$ intervalos iguales, mientras que

d) dentro de cada intervalo, $x$ tiene distribución uniforme.

Los valores esperados de la pérdida de eficiencia, calculada para valores plausibles de $I$ y de $G(60 \leq T \leq 1000 ; 6 \leq G \leq 10)$ parecen fluctuar entre 0.02 y 0.10 .

Además, Cramer afirma apodícticamente: "En la medida en que los intervalos (logarítmicos) sean desiguales, la agrupación será por lo común algo menos eficiente que el sistema que hemos considerado aquí..." 2

Para examinar esta afirmación, invertiremos la pregunta: ¿Cuál agrupación minimiza $S\left(x^{\prime 2}\right)$ ? Van Praag (1970) proporciona una solución, aunque él formuló la pregunta en forma un tanto diferente:

¿Cómo minimizar la pérdida de información que resulta de reemplazar los valores originales de $F(x)$ por las medias de grupo $F\left(\overline{x_{i}}\right)$ ?; $F(x)$ es la función de distribución de $X ; x_{1}, \ldots, x_{n}$ son las fronteras de los grupos, $x_{1}=0$ y $x_{G+1}=\infty$, mientras que $F\left(\bar{x}_{g}\right)=\frac{1}{2}\left[F\left(x_{g}\right)+F\left(x_{g+1}\right)\right]$ y $n$ es una constante.

Dentro de cada grupo, esta pérdida queda definida por

$$
\int_{x_{g}}^{x_{g+1}}\left\{F(x)-F\left(\bar{x}_{g}\right)\right\}^{2} d F(x)
$$

1 Cf. Cramer (1964).

2 Cramer (1964), p. 42. 
Tenemos que encontrar $x_{2}, \ldots, x_{G}$ que minimice:

$$
\text { (A.1.5) } \sum_{g=1}^{G} \int_{x}^{x_{g+1}}\left\{F(x)-F\left(\bar{x}_{g}\right)\right\}^{2} d F(x) \text {, }
$$

o lo que es equivalente, obtener

$$
\begin{gathered}
\text { (A.1.6) el minimo } \stackrel{\sum}{g=1}_{F\left(x_{g}\right)}^{F\left(x_{g+1}\right)}\left\{F(x)-F\left(x_{g}\right)\right\}^{2} d F(x)= \\
=\operatorname{minimo~}_{\left(F\left(x_{2}\right), \ldots, F\left(x_{G}\right)\right)} \sum_{g=1}^{G}\left\{\frac{1}{3}\left(F\left(x_{g+1}\right)-F\left(x_{g}\right)\right)\right\}^{3}
\end{gathered}
$$

Defínase $e_{g}=F\left(x_{g+1}\right)-F\left(x_{g}\right)$, entonces $\sum_{g=1}^{G} e_{g}=1$

Por lo tanto (A.1.6) es equivalente a minimizar $\sum_{g} e_{g}^{3}$ bajo las restricciones $\sum_{\mathrm{g}=1}^{n} e_{\mathrm{g}}=1$ y $e_{\mathrm{g}} \geq 0$

La solución es, desde luego, $e_{g}=n^{-1}$ para $g=1, \ldots, n$, de modo que $F\left(x_{g}\right)=G^{-1}(\mathrm{~g}-1)$.

En vista de que $F(x)$ es la función de distribución de $X$, en nuestro caso el resultado anterior significa que las observaciones deben agruparse de tal modo que todos los casos contengan (aproximadamente) el mismo número de observaciones. Puede demostrarse que la minimización anterior de la pérdida de información es equivalente a minimizar $S\left(x^{\prime 2}\right)$, es decir, la variación intragrupos. La transformación integral de la primera parte de (A.1.6), reemplazando $F(x)$ por $x$, resulta en:

$$
\text { (A.1.7) } \underset{\left(x_{2}, \ldots, x_{G}\right)}{\operatorname{mínimo}} \sum_{i=1}^{G} \int_{x_{g}}^{x_{g+1}}\left(x-\bar{x}_{g}\right)^{2} f(x) d x
$$

donde $f(x)=d F(x) / d x$, esto es, la función de densidad de $x$. Resulta claro que (A.1.7) equivale a minimizar $S\left(x^{\prime 2}\right)$.

\section{APÉNDICE A.2}

ESTIMACIÓN DEL SESGo dEBIDO AL REEMPLAZO dE MEdIAS GEOMÉTRICAS POR MEDIAS ARITMÉTICAS

La diferencia entre los logaritmos de la media geométrica y de la media aritmética depende de la distribución de la variable considerada. Si la variable $x$ tiene distribución lognormal, la diferencia es la mitad de la varianza del logaritmo de la variable: 


\section{(A.2.1) $\quad \log x_{0}^{*}=\log \vec{x}_{0}-\frac{1}{2} \sigma^{2}$,}

donde $x_{0}^{*}=$ media geométrica y $\bar{x}_{0}=$ media aritmética.

Si la variable tiene distribución uniforme, se obtiene:

$$
f(x)=\left(x_{1}-x_{0}\right)^{-1}, \quad x_{0} \leq x \leq x_{1}
$$

y

(A.2.3) $\quad E[x]=\left(x_{1}-x_{0}\right) / 2$

En este último caso, para la distribución de $y=\log x$ se encuentra que:

(A.2.4) $f(y)=\left(x_{1}-x_{0}\right)^{-1} e^{y}, y_{0} \leq y \leq y_{1}$

$$
E[y]=\int_{y_{0}}^{y_{1}}\left(x_{1}-x_{0}\right)^{-1}
$$

y

$$
e^{y} d y=\left(e^{y_{1}}-e^{y_{0}}\right)^{-1}\left[e^{y_{1}}\left(y_{1}-1\right)-e^{y_{0}}\left(y_{0}-1\right)\right]
$$

por lo tanto:

(A.2.6) $\log \bar{x}_{0}-\log x_{0}^{*}=\log \left[\left(x_{1}-x_{0}\right) / 2\right]+$

$$
-\left(e^{y_{1}}-e^{y_{0}}\right)^{-1}\left[e^{y_{1}}\left(y_{1}-1\right)-e^{y_{0}}\left(y_{0}-1\right)\right]
$$

si se establece que:

$$
\text { (A.2.7) } \quad x_{1}=\mu x_{0}
$$

$y$ se sustituye (A.2.7) en (A.2.6) se obtiene:

$$
\text { (A.2.8) } \log x_{0}-\overline{\log } x_{0}^{*}=\log [(\mu-1) / 2]-\{\mu /(\mu-1)\} \log \mu+1
$$

Dependiendo de que $\mu$ tenga un valor de 2 6 1.5, la diferencia será 0.097 y 0.130 , respectivamente.

La aplicación de (A.2.1) a nuestro problema presenta la dificultad de calcular la varianza del logaritmo del gasto total por grupos de ingresos.

Resulta claro que no pueden utilizarse aquí los grupos de ingreso mencionados en la publicación de la encuesta, debido a que nuestra variable determinante no es el ingreso sino el ingreso menos el ahorro, es decir, el gasto total.

Más aún, existe una diferencia sistemática entre las observaciones del ingreso y del gasto total más el ahorro. En los grupos de ingreso reducido, el gasto total es mayor que el ingreso, mientras que en los superiores sucede lo contrario. Por lo tanto, partiendo de los valores conocidos de $\bar{y}_{g}, g=$ $=1, \ldots, G$ ampliamos los intervalos de los logaritmos de los estratos de gasto - "ingreso"-, a medias móviles de parejas de diferencias sucesivas de $\bar{y}_{g}$, 


$$
\left[\left(\bar{y}_{g}-\bar{y}_{g-1}\right)+\left(\bar{y}_{g+1}-\bar{y}_{g}\right)\right] / 2=\left(\bar{y}_{g+1}-\bar{y}_{g-1}\right) / 2
$$

donde $\bar{y}_{g} \equiv \log \bar{x}_{\underline{g}}$, y $\bar{x}_{g}=$ media geométrica de $X$ en el grupo de gasto $g$. Por consiguiente, $y_{g}$ cae a la mitad del estrato del logaritmo del gasto.

Suponiendo que éste abarca el $80 \%$ de las observaciones por grupo de gasto y teniendo en mente que $y$ tiene una distribución normal dentro de cada grupo de gasto, podemos calcular $\mu$ y $\frac{1}{2} \sigma^{2}$ en los grupos de gasto $g=2, \ldots, 8 ; \frac{1}{2} \sigma$ quedaría entre 0.0012 y 0.0034 . Si se escoge el $60 \%$ en lugar del $80 \%$ de las observaciones, la diferencia que resulta no es importante. De lo cual se concluye que cuando se utilizan medias aritméticas como aproximación a las medias geométricas, no hay razón para reestimar los coeficientes y las elasticidades ingreso.

\section{APENDICE B}

CONSECUENCIAS DE LAS CONDICIONES DE SLUTSKY SOBRE LA ESPECIFICACIÓN DE LAS FUNCIONES $f_{k}$ EN EL MODELO DE ASIGNACIÓN DEL GASTO

\section{B.1. Condiciones de primer orden}

Para el propósito que nos ocupa, el modelo de asignación del gasto (2.1.1) se reescribe en forma logarítmica:

(B.1.1) $\quad \ln q_{k}=\ln C-\ln p_{k}+\ln f_{k}-\ln \sum_{h=1}^{K} f_{h}$

en donde se han omitido los argumentos de las funciones para simplificar la exposición.

Lo anterior implica que:

$$
\frac{\partial q_{k}}{\partial p_{l}}=-q_{k} q_{l} C^{-1} p_{l} f_{l}^{-1} \cdot f_{l l}^{\prime}-q_{l}\left(p_{l}^{-1}-f_{l}^{-1} f_{l l}^{\prime}\right) \delta_{k l}
$$

y que

$$
q_{l} \frac{\partial q_{k}}{\partial C}=q_{k} q_{l}\left[C^{-1}+f_{k}^{1} f_{k C}^{\prime}-\left(\sum_{h} f_{h}\right)^{-1}\left(\sum_{h} f_{h C}^{\prime}\right)\right],
$$

donde $\quad f_{l l}^{\prime}=\partial f_{l} / \partial q_{l} \quad$ y $f_{k C}^{\prime}=\partial f_{k} / \partial C$.

Al intercambiar $k$ y $l$, se obtiene:

$$
\frac{\partial q_{k}}{\partial p_{l}}-\frac{\partial q_{l}}{\partial p_{k}}=-q_{k} q_{l} C^{-1}\left(p_{l} f_{l}^{-1} f_{l l}^{\prime}-p_{k} f_{k}^{-1} f_{k k}^{\prime}\right)
$$

y

$$
q_{l} \frac{\partial q_{k}}{\partial p_{k}}-q_{k} \frac{\partial q_{l}}{\partial C}=q_{k} q_{l}\left(f_{k}^{-1} f_{k C}^{\prime}-f_{l}^{1} f_{l C}^{\prime}\right)
$$


Dado que las condiciones de Slutsky (2.1.3 a 2.1.5) implican que los elementos en (B.1.4) y (B.1.5) deben ser opuestos entre sí, es decir, sumar cero, se obtiene:

$$
p_{i}^{-1} f_{l l}^{\prime}+C f_{l}^{-1} f_{l C}^{\prime}=p_{k} f_{k}^{-1} f_{k k}^{\prime}+C f_{k}^{-1} f_{k c}^{\prime}
$$

lo cual es equivalente a $(2.2 .1 .6)$, ya que

$$
p_{i} f_{l}^{1} f_{l l}^{\prime}=\frac{\partial \ln f_{l}}{\partial \ln p_{l}}
$$

$\mathrm{y}$

$$
C f_{l}^{-1} f_{l C}^{\prime}=\frac{\partial \ln f_{l}}{\partial \ln C}
$$

B.2. Condiciones de segundo orden

Si $f_{k}\left(p_{k} / C\right)$ se especifica como en (2.1.4), que resulta de la ecuación (2.1.5) para $q_{k}$, se encuentra que:

(B.2.1) $\frac{\partial q_{k}}{\partial p_{l}}=-\left(1-\alpha_{l}\right) q_{i} p_{l}^{-1} \delta_{k l}-\alpha_{l} C^{-1} q_{k} q_{l}$

y

(B.2.2) $\frac{\partial q_{k}}{\partial C}=\left(1-\alpha_{k}+\bar{\alpha}\right) C^{-1} q_{k}$

donde $\quad \bar{\alpha}=\sum_{h=1}^{K} \alpha_{h} w_{h}$

por lo tanto:

$$
s_{k l}=\left\{\left(1-\alpha_{k}-\alpha_{l}+\bar{\alpha}\right)-w_{l}^{-1}\left(1-\alpha_{l}\right) \delta_{k l}\right\} C^{-1} q_{k} q_{l}=s_{l k}
$$

Para examinar $S$ y ver la posibilidad de que sea negativa semidefinida, las expresiones B.2.1 a B.2.3 se reescriben ahora en notación matricial:

$$
Q_{p}=-\dot{q} \dot{p}^{-1}(I-\dot{\alpha})-C^{-1} q q^{\prime} \dot{\alpha}
$$

$$
q_{c}=C^{-1}[(1+\bar{\alpha}) I-\dot{\alpha}] q
$$

y

$$
\begin{aligned}
S & =Q_{p}+q_{c} q^{\prime} \\
& =C^{-1}\left[(I+\dot{\bar{\alpha}}) q q^{\prime}-\dot{\alpha} q q^{\prime}-q q^{\prime} \dot{\alpha}\right]-\dot{p}^{-1}(I-\dot{\alpha}) \dot{q}
\end{aligned}
$$

Con un vector arbitrario $z$ de $K$ elementos escribimos entonces:

$$
z^{\prime} S z=C\left[-z^{\prime}(I-\dot{\bar{\alpha}}) \dot{p}^{-1} w w^{\prime} \dot{p}^{-1} z\right.
$$




$$
\begin{gathered}
+z^{\prime}(I-\dot{\alpha}) \dot{p}^{-1} w w^{\prime} \dot{p}^{-1} z \\
+z^{\prime} \dot{p}^{-1} w w^{\prime} \dot{p}^{-1}(I-\dot{\alpha}) z \\
\left.+z^{\prime} \dot{p}^{-1}(I-\dot{\alpha}) \dot{w} \dot{p}^{-1} z\right] \\
=C\left[-x^{\prime}(I-\dot{\bar{\alpha}}) w w^{\prime} x+2 x^{\prime}(I-\dot{\alpha}) w w^{\prime} x\right. \\
\left.\quad-x^{\prime}(I-\dot{\alpha}) \dot{w} x\right]
\end{gathered}
$$

en donde $\mathrm{x}=\dot{p}^{-1} z$

$$
=C\left[-\bar{x}^{2}(1-\bar{\alpha})+2 \bar{x} x^{\prime}(I-\dot{\alpha}) w-x^{\prime}(I-\dot{\alpha}) \dot{w} x\right]
$$

donde $\bar{x}=w^{\prime} x$

$$
=C\left[(x-\bar{x})^{\prime} \dot{w}(I-\dot{\alpha})(x-\bar{x})\right],
$$

y $\imath^{\prime}=\{1, \ldots, 1\}$ es un vector de suma de tamaño $K$. En vista de que

$$
\begin{aligned}
& \overline{x^{2}}(1-\bar{\alpha})=(\bar{x} \iota)^{\prime} \dot{w}(I-\dot{\alpha})(\bar{x} \iota), \\
& \overline{2 x} x^{\prime}(I-\dot{\alpha}) w=2(\bar{x} b)^{\prime} \dot{w}(I-\dot{\alpha}) x
\end{aligned}
$$

y

$$
x^{\prime}(I-\dot{\alpha}) \dot{w} x=x^{\prime} \dot{w}(I-\dot{\alpha}) x
$$

Por lo tanto:

(B.2.8) $z^{\prime} S z<0$ si $\alpha<$, es decir $\alpha_{k}<1$ para $k=1, \ldots, K$ quería.

La igualdad ocurre si y sólo si $x=\imath$, es decir, si $z=p$ como se re-

\section{APENDICE C}

Dependencia del PRECIO PROMEdIO DE UN BIEN HETEROGÉNeO SOBRE EL GASTO TOTAL Y EL TAMAÑo DEL HOGAR

\section{C.1. Relación entre el precio promedio y el gasto total}

Supóngase que varios rubros presupuestales relacionados entre sí se combinan para dar un bien compuesto $s$; entonces su precio promedio $p_{s}$ se define como:

(C.1.1) $\quad p_{s}=q_{s}^{-1} \sum_{k \varepsilon\{s\}} p_{k} q_{k}$,

donde $q_{s}=\sum_{k \varepsilon\{s\}} q_{k}$, suponiendo que no solamente es permisible sumar las 
$q_{k}$ para todas las $k \varepsilon\{s\}$ por estar expresadas en las mismas unidades, sino también porque la agregación tiene algún significado.

La sensibilidad de $p_{s}$ respecto a cambios en el gasto total $C$ puede ex presarse mediante:

$$
\begin{aligned}
E\left(p_{s}, C\right) & =C p_{s}^{-1} \frac{\partial p_{s}}{\partial C} \\
& =w_{s}^{-1}\left[\sum_{k \varepsilon\{s\}} p_{k} \frac{\partial q_{k}}{\partial C}-p_{s} \frac{\partial q_{s}}{\partial C}\right] \\
& =w_{s}^{-1} \sum_{k \varepsilon\{s\}}\left(p_{k}-p_{s}\right) \frac{\partial q_{k}}{\partial C} \\
& =C_{s}^{-1} \sum_{k \varepsilon\{s\}} q_{k}\left(p_{k}-p_{s}\right) E\left(q_{k}, C\right) \\
& =C_{s}^{-1} \sum_{k \varepsilon\{s\}} q_{k}\left(p_{k}-p_{s}\right)\left\{E\left(q_{k}, C\right)-E\left(q_{s}, C\right)\right\}
\end{aligned}
$$

en virtud de (C.1.1), se implica que

$$
\sum_{k \varepsilon\{s\}} q_{k}\left(p_{k}-p_{s}\right) \equiv 0
$$

Aparte de un factor multiplicativo (C.1.2) expresa la elasticidad "cualitativa" $E\left(p_{s}, C\right)$ como una especie de covarianza entre los precios $p_{k}$ y las elasticidades ingreso $E\left(q_{k}, C\right)$, ponderadas con las cantidades $q_{k}$, para todas las $k \varepsilon\{s\}$.

En general, puede esperarse que las elasticidades ingreso sean mayores a medida que los bienes del subconjunto sean más caros. En consecuencia:

(C.1.3) $E\left(p_{s}, C\right)>0$ en general.

En particular, el modelo de asignación del gasto (2.1.5) produce: (C.1.4) $\quad E\left(p_{s}, C\right)=-C_{s}^{-1} \sum_{k \varepsilon\{s\}} q_{k}\left(p_{k}-p_{s}\right)\left(\alpha_{k}-\alpha_{s}\right)>0$

en virtud de (2.2.1) y (C.1.1) si es que hemos de esperar algún paralelismo entre carestía (según $p_{k}$ sea mayor) y lujo (según $\alpha_{k}$ sea menor).

C.2. Relación entre precio promedio y tamaño del hogar

De acuerdo con (1.1.1) obtenemos una relación aproximada para $\Delta \log p_{s}$, esto es, el cambio relativo en el precio $p_{s}$, del bien compuesto, como resultado de aumentar el número de miembros en el hogar en el grupo $g\left(n_{g}\right)$ en una unidad; esta relación es:

$$
\begin{aligned}
n_{g} \Delta \log p_{s} & =n_{g} p_{s}^{-1} \Delta p_{s} \\
& \approx n_{s} p_{s}^{-1}\left\{q_{s}^{-1} \sum_{k \varepsilon\{s\}} p_{k} \Delta q_{k}-p_{s} \Delta q_{s}\right. \\
& \approx n_{g} C_{s}^{-1} \sum_{k \varepsilon\{s\}} q_{k}\left(p_{k}-p_{s}\right) \Delta \log q_{k}
\end{aligned}
$$


Para el modelo de asignación del gasto (2.1.5), el efecto de un aumento $\Delta n_{g}=1$ sobre el aumento relativo en el consumo de $q_{k}$ está expresado por:

$$
n_{g} \Delta \log q_{k}=v_{k g} \alpha_{k} \sum_{h} w_{h} \nu_{h g} \alpha_{h}
$$

donde $\quad v_{k g}=\frac{e_{k g} n_{g}}{\sum_{g^{\prime}} e_{k g^{\prime}} n_{g}}=e_{k g} n_{g} / m_{k}$

igual a la participación del grupo $g$ en el hogar con respecto al consumo de $k$. Al sustituir (C.2.2) en (C.2.1) se obtiene:

$$
n_{g} \Delta \log p_{s} \approx C_{s}^{-1} \sum_{k \varepsilon\{s\}} q_{k}\left(p_{k}-p_{s}\right)\left(\nu_{k g} \alpha_{k}-\overline{\nu_{h g} \alpha_{h}}\right)
$$

donde

$$
\overline{\nu_{h g} \alpha_{h}}=\underset{h \varepsilon\{s\}}{\sum} w_{h} \nu_{h g} \alpha_{h}
$$

Este último resultado se parece a (C.1.4), salvo por el signo y la multiplicación de $\alpha_{k}$ por los $\nu_{k g}$. Suponiendo que no existe correlación negativa entre los $\nu_{h g}$ y las $\alpha_{h}$ para las $h \varepsilon\{s\}$, es decir, una correlación positiva o-lo que es más probable- una correlación nula con la $\alpha_{k}$, el término de suma $\sum_{k \varepsilon\{s\}}$ representa de nuevo una covarianza negativa (ponderada).

La conclusión es que, en general, un aumento en el tamaño del hogar (debido a un aumento en un grupo por sexo y edad), si todo lo demás permanece igual (entre otras cosas el ingreso familiar), ocasionará un desplazamiento del consumo de los bienes más caros hacia los más baratos, esto es, una reducción del precio promedio del bien compuesto. Esto es razonable, ya que un aumento en el tamaño del hogar (ceteris paribus) implica una disminución equivalente en el ingreso real de cada consumidor estándar.

\section{REFERENCIAS BIBLIOGRÁFICAS}

Banco de México (1963), Oficina de Estudios sobre Proyecciones Agrícolas, Encuesta sobre ingresos y gastos familiares en México.

J. Blokland, y W. H. Somermeyer (1970), "Effects of Family Size and Composition on Family Expenditure According to an Allocation Model", Informe 7020 del Instituto de Econometría de Holanda, Rotterdam.

I. S. Cramer (1964), "Efficient Grouping, Regression and Correlation in Engel Curve Analysis", Journal of the American Statistical Association, Vol. 59, pp. 233-250.

H. S. Houthakker (1960), "Additive Preference", Econometrica, Vol. 28, pp. 244257 .

G. J. A. Mensink (1972), "Het National Budgetonderzoek 1963/'65: een analyse van de uitkomsten" (en holandés con resumen en inglés: "The National Family Budget Survey 1963/'65: an Analysis of the Results"), Statistische en Econometrische Onderzoekingen (Estudios estadísticos y econométricos), Núm. 12, editado por el Departamento Central de Estadística de Holanda. 
Van Praag B. M. S. (1970), "The Welfare Function of Income in Belgium: an Emirical Investigation", European Economic Review, Vol. 2, pp. 337-369.

S. J. Prais, y J. Aithchison (1954), "The Grouping of Observations in Regression Analysis", Review of the Int. Stat. Institute, Vol. 22.

S. J. Prais, y H. S. Houthakker (1955), The Analysis of Family Budgets, Cambridge: Cambridge University Press.

W. H. Somermeyer, J. G. M. Hilhorst y J. W. W. A. (1961), “A Method for Estimation Price and Income Elasticities from Time Series and its Application to Consumers Expenditures in the Netherlands, 1949-1959", Statistical Studies, Núm. 13, pp. 30-53, editado por el Departamento Central de Estadística de Holanda.

H. Theil (1951), "Qualities, Prices and Budget Enquiries", The Review of Economic Studies, Vol. 19, pp. 129-147.

H. Theil y Mnookin (1966), "The Information Value of Demand Equations and Predictions", The Journal of Political Economy, Vol. 74, pp. 34-45. 